\title{
PATH-FOLLOWING METHODS FOR A CLASS OF CONSTRAINED MINIMIZATION PROBLEMS IN FUNCTION SPACE*
}

\author{
MICHAEL HINTERMÜLLER ${ }^{\dagger}$ AND KARL KUNISCH ${ }^{\ddagger}$
}

\begin{abstract}
Path-following methods for primal-dual active set strategies requiring a regularization parameter are introduced. Existence of a primal-dual path and its differentiability properties are analyzed. Monotonicity and convexity of the primal-dual path value function are investigated. Both feasible and infeasible approximations are considered. Numerical path-following strategies are developed and their efficiency is demonstrated by means of examples.
\end{abstract}

Key words. semismooth Newton methods, path-following methods, active set strategy, primaldual methods

AMS subject classifications. 49M15, 49M37, 65K05, 90C33

DOI. $10.1137 / 040611598$

1. Introduction. Primal-dual active set strategies or, in some cases equivalently, semismooth Newton methods, were proved to be efficient methods for solving constrained variational problems in function space $[1,9,10,11,12,13]$. In certain cases regularization is required, resulting in a family of approximating problems with more favorable properties than those of the original one, [12, 13]. In previous work [13] convergence, and in some cases rate of convergence, with respect to the regularization parameter was proved. In the numerical work the adaptation of these parameters was heuristic, however. The focus of the present investigation is on an efficient control of the regularization parameter in the primal-dual active set strategy for a class of constrained variational problems. To explain the involved issues we proceed mostly formally in this section and consider the problem

$$
\left\{\begin{array}{l}
\min \mathcal{J}(v) \quad \text { over } v \in X \\
\text { s.t. } G v \leq \psi,
\end{array}\right.
$$

where $\mathcal{J}$ is a quadratic functional on a Hilbert space $X$, and $G: X \rightarrow Y$. It is assumed that $Y \subset L^{2}(\Omega)$ is a Hilbert lattice with ordering $\leq$ induced by the natural ordering of $L^{2}(\Omega)$. We note that (1) subsumes problems of very different nature. For example, for the control constrained optimal control problem

$$
\left\{\begin{array}{l}
\min \frac{1}{2}|y-z|_{L^{2}}^{2}+\frac{\alpha}{2}|u|_{L^{2}}^{2} \\
\text { s.t. }-\Delta y=u \text { in } \Omega, \quad y=0 \text { on } \partial \Omega \\
u \leq \psi \text { a.e. in } \Omega
\end{array}\right.
$$

with $\Omega$ a bounded domain in $\mathbb{R}^{n}, z \in L^{2}(\Omega), \alpha>0$, one can use $y=(-\Delta)^{-1} u$, where $\Delta$ denotes the Laplacian with homogenous Dirichlet boundary conditions, and arrive

${ }^{*}$ Received by the editors July 14, 2004; accepted for publication (in revised form) November 21, 2005; published electronically May 3, 2006. This research was partially supported by the Fonds zur Förderung der wissenschaftlichen Forschung under SFB 03 "Optimierung und Kontrolle."

http://www.siam.org/journals/siopt/17-1/61159.html

${ }^{\dagger}$ Department of Computational and Applied Mathematics, Rice University, Houston, TX.

$\ddagger$ Institute of Mathematics and Scientific Computing, University of Graz, Graz, Austria (michael. hintermueller@uni-graz.at, karl.kunisch@uni-graz.at). 
at

$$
\left\{\begin{array}{l}
\min \frac{1}{2}\left|(-\Delta)^{-1} u-z\right|^{2}+\frac{\alpha}{2}|u|^{2} \\
\text { s.t. } u \leq \psi \text { a.e. in } \Omega
\end{array}\right.
$$

which is clearly of the form (1). For $\mathcal{J}(v)=\frac{1}{2} \int_{\Omega}|\nabla v|^{2} d x-\int_{\Omega} f v, X=H_{0}^{1}(\Omega)$, and $G=I$ we obtain the classical obstacle problem. For state constrained control problems with $y \leq \psi$ one has

$$
\left\{\begin{array}{l}
\min \frac{1}{2}\left|(-\Delta)^{-1} u-z\right|^{2}+\frac{\alpha}{2}|u|^{2} \\
\text { s.t. }(-\Delta)^{-1} u \leq \psi \text { a.e. in } \Omega
\end{array}\right.
$$

which is also of the form (1). From the point of view of duality theory these three problems are very different. While it is straightforward to argue the existence of a Lagrange multiplier in $L^{2}(\Omega)$ for the control constrained optimal control problem, it is already more involved and requires additional assumptions to guarantee its existence in $L^{2}(\Omega)$ for obstacle problems, and for state constrained problems the Lagrange multiplier is only a measure. If we resort to a formal discussion, then in either of these cases we arrive at the optimality system of the form

$$
\left\{\begin{array}{l}
\mathcal{J}^{\prime}(v)+G^{*} \lambda=0 \\
\lambda=\max (0, \lambda+c(G(v)-\psi))
\end{array}\right.
$$

for any fixed $c>0$. Here, $G^{*}$ denotes the adjoint of $G$. The second equation in (2) is equivalent to $\lambda \geq 0, G(v) \leq \psi$, and $\lambda(G(v)-\psi)=0$.

Continuing formally, the primal-dual active set strategy determines the active set at iteration level $k$ by means of

$$
\mathcal{A}_{k+1}=\left\{x \in \Omega: \lambda_{k}(x)+c\left(G\left(v_{k}\right)(x)-\psi(x)\right)>0\right\},
$$

assigns the inactive set $\mathcal{I}_{k+1}=\Omega \backslash \mathcal{A}_{k+1}$, and updates $(v, \lambda)$ by means of

$$
\left\{\begin{array}{l}
\mathcal{J}^{\prime}\left(v_{k+1}\right)+G^{*} \lambda_{k+1}=0, \\
\lambda_{k+1}=0 \text { on } \mathcal{I}_{k+1}, \quad\left(G\left(v_{k+1}\right)-\psi\right)(x)=0 \text { for } x \in \mathcal{A}_{k+1} .
\end{array}\right.
$$

These auxiliary problems require special attention. For obstacle problems the constraint $v_{k+1}=\psi$ on $\mathcal{A}_{k+1}$ induces that the associated Lagrange multiplier $\lambda_{k+1}$ is in general less regular than the Lagrange multiplier associated with $v \leq \psi$ for the original problem; see, e.g., [13]. For problems with combined control and state constraints it may happen that due to the assignment on $\mathcal{I}_{k+1}$ and $\mathcal{A}_{k+1},(3)$ has no solution while the original problem does. For these reasons in, e.g., $[9,12,13]$ the second equation in (2) was regularized, resulting in the family of equations

$$
\left\{\begin{array}{l}
\mathcal{J}^{\prime}(v)+G^{*} \lambda=0 \\
\lambda=\max (0, \bar{\lambda}+\gamma(G(v)-\psi))
\end{array}\right.
$$

where $\bar{\lambda}$ is fixed, possibly $\bar{\lambda}=0$, and $\gamma \in \mathbb{R}^{+}$. In the above-mentioned references it was shown that under appropriate conditions the solutions $\left(v_{\gamma}, \lambda_{\gamma}\right)$ to $(4)$ exist, the quantity $\lambda_{\gamma}$ enjoys extra regularity, and $\left(v_{\gamma}, \lambda_{\gamma}\right)$ converge to the solution of (2) as $\gamma \rightarrow \infty^{+}$. 
In previous numerical implementations the increase of $\gamma$ to infinity was heuristic. As the system (4) becomes increasingly ill-conditioned as $\gamma$ tends to $\infty$, in this paper a framework for a properly controlled increase of $\gamma$-values will be developed in order to cope with the conditioning problem. In fact, in a typical algorithmic regime for solving (1) one uses the solution $\left(v_{\gamma}, \lambda_{\gamma}\right)$ to (4) for some $\gamma$ as the initial guess for the solution to (4) for the updated $\gamma$-value $\gamma^{+}>\gamma$. Typically, if $\gamma^{+} \gg \gamma$, then $\left(v_{\gamma}, \lambda_{\gamma}\right)$ is only a poor approximation of $\left(v_{\gamma^{+}}, \lambda_{\gamma^{+}}\right)$, which in addition to numerical linear algebra issues (like ill-conditioned system matrices) causes severe stability problems for iterative solvers for (4) such as semismooth Newton methods. Together with developing a new $\gamma$ update strategy, we aim at solving the auxiliary problems (4) only inexactly to keep the overall computational cost low. To this end we define neighborhoods of the path which allow inexact solutions and which contract in a controlled way towards the path as the iteration proceeds. Our work is inspired by concepts from path-following methods in finite dimensional spaces $[4,5,16,18,19]$. We first guarantee the existence of a sufficiently smooth path $\gamma \rightarrow\left(v_{\gamma}, \lambda_{\gamma}\right)$, with $\gamma \in(0, \infty)$ in appropriately chosen function spaces. Once the path is available it can be used as the basis for updating strategies of the path parameter. Given a current value $\gamma_{k}$, with associated primal and dual states $\left(v_{\gamma_{k}}, \lambda_{\gamma_{k}}\right)$, the $\gamma$-update should be sufficiently large to make good progress towards satisfying the complementarity conditions. On the other hand, since we are not solving the problems along the path exactly, we have to use safeguards against steps which would lead us too far off the path. Of course, these goals are impeded by the fact that the path is not available numerically. To overcome this difficulty we use qualitative properties of the value function, like monotonicity and convexity, which can be verified analytically. These suggest the introduction of model functions which will be shown to approximate the value functional along the path very well. We use these model functions for our updating strategies of $\gamma$. In the case of exact path-following we can even prove convergence of the resulting strategy. In the present paper the program just described is carried out for a class of problems corresponding to contact problems. State constrained optimal control problems require a different approach that will be considered independently. As we shall see, the (infinite dimensional) parameter $\bar{\lambda}$ can be used to guarantee that the iterates of the primal variable are feasible. Further, it turns out that the numerical behavior of infeasible approximations is superior to the feasible ones from the point of view of iteration numbers.

Interior point methods also require an additional parameter, which, however, enters into (2) differently. For the problem under consideration here, the interiorpoint relaxation replaces the second equation in (2) by

$$
\lambda(x)(\psi-G(v))(x)=\frac{1}{\gamma} \quad \text { for } x \in \Omega .
$$

Path-following interior-point methods typically start strictly feasible, with iterates which are required to stay strictly feasible during the iterations while satisfying, or satisfying approximately, the first equation in (2) and (5). Path-following interiorpoint methods have not received much attention for infinite dimensional problems yet. In fact, we are aware of only [17], where such methods are analyzed for optimal control problems related to ordinary differential equations. For the problem classes that we outlined at the beginning of this section, the primal-dual active set strategy proved to be an excellent competitor to interior-point methods, as was demonstrated, for example, in [1] comparing these two methods.

This paper is organized as follows. Section 2 contains the precise problem formulation and the necessary background on the primal-dual active set strategy. The 
existence and regularity of the primal-dual path is discussed in section 3. Properties of the primal-dual path value functional are analyzed in section 4 . Section 5 contains the derivation of the proposed model functions for the primal-dual path value functional. Exact as well as inexact path-following algorithms are proposed in section 6, and their numerical behavior is discussed there as well.

2. Problem statement, regularization, and its motivation. We consider

$$
\left\{\begin{array}{l}
\min \frac{1}{2} a(y, y)-(f, y) \quad \text { over } \quad y \in H_{0}^{1}(\Omega) \\
\text { s.t. } y \leq \psi
\end{array}\right.
$$

where $f \in L^{2}(\Omega), \psi \in H^{1}(\Omega)$, with $\psi_{\mid \partial \Omega} \geq 0$, where $\Omega$ is a bounded domain in $\mathbb{R}^{n}$ with Lipschitz continuous boundary $\partial \Omega$. Throughout, $(\cdot, \cdot)$ denotes the standard $L_{2}(\Omega)$-inner product, and we assume that $a(\cdot, \cdot)$ is a bilinear form on $H_{0}^{1}(\Omega) \times H_{0}^{1}(\Omega)$ satisfying

$$
a(v, v) \geq \nu|v|_{H_{0}^{1}}^{2} \text { and } a(w, z) \leq \mu|w|_{H^{1}}|z|_{H^{1}}
$$

for some $\nu>0, \mu>0$ independent of $v \in H_{0}^{1}(\Omega)$ and $w, z \in H^{1}(\Omega)$. Here and throughout we use $|v|_{H_{0}^{1}}=|\nabla v|_{L^{2}}$ for $v \in H_{0}^{1}(\Omega)$, which defines a norm on $H_{0}^{1}(\Omega)$ due to Friedrichs' inequality, and $|w|_{H^{1}}=\left(|w|_{L^{2}}^{2}+|\nabla w|_{L^{2}}^{2}\right)^{1 / 2}$ denotes the standard $H^{1}$-norm; see, e.g., [2]. Moreover, let $A: H_{0}^{1}(\Omega) \rightarrow H^{-1}(\Omega)$ be defined by

$$
a(v, w)=\langle A v, w\rangle_{H^{-1}, H_{0}^{1}} \quad \text { for all } v, w \in H_{0}^{1}(\Omega) .
$$

It is well known that $(\mathrm{P})$ admits a unique solution $y^{*} \in H_{0}^{1}(\Omega)$ with associated Lagrange multiplier $\lambda^{*}=-A y^{*}+f$, satisfying the optimality system

$$
\left\{\begin{array}{l}
a\left(y^{*}, v\right)+\left\langle\lambda^{*}, v\right\rangle_{H^{-1}, H_{0}^{1}}=(f, v), \\
\left\langle\lambda^{*}, y^{*}-\psi\right\rangle_{H^{-1}, H_{0}^{1}}=0, \quad y^{*} \leq \psi,\left\langle\lambda^{*}, v\right\rangle \leq 0 \text { for all } v \leq 0 .
\end{array}\right.
$$

This also holds with $f \in H^{-1}(\Omega)$. Under well-known additional requirements on $a, \psi$, and $\Omega$, as for example

$$
\begin{cases}a(v, w)=\int_{\Omega}\left(\sum a_{i j} v_{x_{i}} w_{x_{j}}+d v w\right), & \text { with } a_{i j} \in C^{1}(\bar{\Omega}), d \in L^{\infty}(\Omega), \\ d \geq 0, \quad \psi \in H^{2}(\Omega), \partial \Omega \quad \text { is } C^{1,1}, & \text { or } \Omega \text { is a convex polyhedron, }\end{cases}
$$

we have $\left(y^{*}, \lambda^{*}\right) \in H^{2}(\Omega) \times L^{2}(\Omega)$, and the optimality system can be expressed as

$$
\left\{\begin{array}{l}
A y^{*}+\lambda^{*}=f \text { in } L^{2}(\Omega) \\
\lambda^{*}=\left(\lambda^{*}+c\left(y^{*}-\psi\right)\right)^{+} \text {for some } c>0
\end{array}\right.
$$

where $(v)^{+}=\max (0, v)$; for details see, e.g., [14].

Our aim is the development of Newton-type methods for solving (7) or (9), which is complicated by the system of inequalities in (7) and the nondifferentiable maxoperator in (9). In the recent past significant progress was made in the investigation of semismooth Newton methods and primal-dual active set methods for coping with nondifferentiable functionals in infinite dimensional spaces; see, for instance, [10, 15]. A direct application of these techniques to (9) results in the following algorithm. 


\section{Algorithm A.}

(i) Choose $c>0,\left(y_{0}, \lambda_{0}\right)$; set $k=0$.

(ii) Set $\mathcal{A}_{k+1}=\left\{x \in \Omega: \lambda_{k}(x)+c\left(y_{k}(x)-\psi(x)\right)>0\right\}$.

(iii) Compute $y_{k+1}=\arg \min \left\{\frac{1}{2} a(y, y)-(f, y): y=\psi\right.$ on $\left.\mathcal{A}_{k+1}\right\}$.

(iv) Let $\lambda_{k+1}$ be the Lagrange multiplier associated with the constraint in (iii), with $\lambda_{k+1}=0$ on $\Omega \backslash \mathcal{A}_{k+1}$.

(v) Set $k:=k+1$ and go to (ii).

The optimality system for the variational problem in (iii) is given by

$$
\left\{\begin{array}{l}
a\left(y_{k+1}, v\right)+\left\langle\lambda_{k+1}, v\right\rangle_{H^{-1}, H_{0}^{1}}=(f, v) \text { for all } v \in H_{0}^{1}(\Omega), \\
y_{k+1}=\psi \text { on } \mathcal{A}_{k+1}, \quad \lambda_{k+1}=0 \text { on } \mathcal{I}_{k+1}=\Omega \backslash \mathcal{A}_{k+1} .
\end{array}\right.
$$

This corresponds to (3) in our introductory discussion. The Lagrange multiplier associated with the constraint $y=\psi$ on $\mathcal{A}_{k+1}$ is in general only a distribution in $H^{-1}(\Omega)$ and is not in $L^{2}(\Omega)$. In fact, $\lambda_{k+1}$ is related to the jumps in the normal derivatives of $y$ across the interface between $\mathcal{A}_{k+1}$ and $\mathcal{I}_{k+1}$ [13]. This complicates the convergence analysis for Algorithm A since the calculus of Newton (or slant) differentiability [10] does not apply. We note that these difficulties are not present if (7) or (9) is discretized. However, they are crucial for the treatment of infinite dimensional problems, and as such they are generic. Analogous difficulties arise for state constrained optimization problems, for inverse problems with BV-regularization, and for elasticity problems with contract and friction, to mention a few. This suggests the introduction of regularized problems, which in our case are chosen as

$\left(P_{\gamma}\right) \quad \min \frac{1}{2} a(y, y)-(f, y)+\frac{1}{2 \gamma} \int_{\Omega}\left|(\bar{\lambda}+\gamma(y-\psi))^{+}\right|^{2} \quad$ over $y \in H_{0}^{1}(\Omega)$,

where $\gamma>0$ and $\bar{\lambda} \in L^{2}(\Omega), \bar{\lambda} \geq 0$ are fixed. For later use we denote the objective functional of $\left(P_{\gamma}\right)$ by $J(y ; \gamma)$. The choice of $\bar{\lambda}$ will be used to influence the feasibility of the solution $y_{\gamma}$ of $\left(P_{\gamma}\right)$. Using Lebesgue's bounded convergence theorem to differentiate the max under the integral in $J(y ; \gamma)$, the first order optimality condition associated with $\left(P_{\gamma}\right)$ is given by

$$
\left\{\begin{array}{l}
a\left(y_{\gamma}, v\right)+\left(\lambda_{\gamma}, v\right)=(f, v) \text { for all } v \in H_{0}^{1}(\Omega), \\
\lambda_{\gamma}=\left(\bar{\lambda}+\gamma\left(y_{\gamma}-\psi\right)\right)^{+}
\end{array}\right.
$$

where $\left(y_{\gamma}, \lambda_{\gamma}\right) \in H_{0}^{1}(\Omega) \times L^{2}(\Omega)$. With (8) holding, we have $y_{\gamma} \in H^{2}(\Omega)$. The primaldual active set strategy, or equivalently the semismooth Newton method, for $\left(P_{\gamma}\right)$ is given next. For its statement and for later use we introduce $\chi_{\mathcal{A}^{k+1}}$, the characteristic function of the set $\mathcal{A}^{k+1} \subseteq \Omega$.

Algorithm B.

(i) Choose $\bar{\lambda} \geq 0,\left(y_{0}, \lambda_{0}\right)$; set $k=0$.

(ii) Set $\mathcal{A}_{k+1}=\left\{x \in \Omega: \bar{\lambda}(x)+\gamma\left(y_{k}(x)-\psi(x)\right)>0\right\}, \mathcal{I}_{k+1}=\Omega \backslash \mathcal{A}_{k+1}$.

(iii) Solve for $y_{k+1} \in H_{0}^{1}(\Omega)$ : $a\left(y_{k+1}, v\right)+\left(\left(\bar{\lambda}+\gamma\left(y_{k+1}-\psi\right)\right) \chi_{\mathcal{A}_{k+1}}, v\right)=(f, v)$ for all $v \in H_{0}^{1}(\Omega)$.

(iv) Set

$$
\lambda_{k+1}= \begin{cases}0 & \text { on } \mathcal{I}_{k+1}, \\ \bar{\lambda}+\gamma\left(y_{k+1}-\psi\right) & \text { on } \mathcal{A}_{k+1} .\end{cases}
$$


Algorithm B was analyzed in [13], where global as well as locally superlinear convergence for every fixed $\gamma>0$ were established. However, the choice and adaptation (increase) of $\gamma$ was heuristic in [13] and earlier work. The focus of the present investigation is the automatic adaptive choice of $\gamma$. We shall utilize the following two results, which we recall from [13] where the proofs can also be found.

Proposition 2.1. The solutions $\left(y_{\gamma}, \lambda_{\gamma}\right)$ to $\left(\mathrm{OC}_{\gamma}\right)$ converge to $\left(y^{*}, \lambda^{*}\right)$ in the sense that $y_{\gamma} \rightarrow y^{*}$ strongly in $H_{0}^{1}(\Omega)$ and $\lambda_{\gamma} \rightarrow \lambda^{*}$ weakly in $H^{-1}(\Omega)$ as $\gamma \rightarrow \infty$.

We say that $a$ satisfies the weak maximum principle if for any $v \in H_{0}^{1}(\Omega)$

$$
a\left(v, v^{+}\right) \leq 0 \text { implies } v^{+}=0 .
$$

Proposition 2.2. Assume that (11) holds and let $0<\gamma_{1} \leq \gamma_{2}<\infty$.

(a) In the infeasible case, i.e., for $\bar{\lambda}=0$, we have $y^{*} \leq y_{\gamma_{2}} \leq y_{\gamma_{1}}$.

(b) In the feasible case, i.e., if

$$
\bar{\lambda} \geq 0 \text { and }\langle\bar{\lambda}-f+A \psi, v\rangle_{H^{-1}, H_{0}^{1}} \geq 0 \text { for all } v \in H_{0}^{1}(\Omega),
$$

with $v \geq 0$, then $y_{\gamma_{1}} \leq y_{\gamma_{2}} \leq y^{*} \leq \psi$.

3. The primal-dual path. In this section we introduce the primal-dual path and discuss its smoothness properties.

Definition 3.1. The family of solutions $\mathcal{C}=\left\{\left(y_{\gamma}, \lambda_{\gamma}\right): \gamma \in(0, \infty)\right\}$ to $\left(\mathrm{OC}_{\gamma}\right)$, considered as subset of $H_{0}^{1}(\Omega) \times H^{-1}(\Omega)$, is called the primal-dual path associated with $(\mathrm{P})$.

For $r \geq 0$ we further set $\mathcal{C}_{r}=\left\{\left(y_{\gamma}, \lambda_{\gamma}\right): \gamma \in[r, \infty)\right\}$, and with some abuse of terminology we also refer to $\mathcal{C}_{r}$ as a path. In the following lemma we denote by $\hat{y}$ the solution to the unconstrained problem

$$
\min J(y)=\frac{1}{2} a(y, y)-(f, y) \quad \text { over } y \in H_{0}^{1}(\Omega) .
$$

Subsequently, in connection with convergence of a sequence in function space we use the subscript "weak" together with the space to indicate convergence in the weak sense.

Lemma 3.2. For each $r>0$ the path $\mathcal{C}_{r}$ is bounded in $H_{0}^{1}(\Omega) \times H^{-1}(\Omega)$, with $\lim _{\gamma \rightarrow \infty}\left(y_{\gamma}, \lambda_{\gamma}\right)=\left(y^{*}, \lambda^{*}\right)$ in $H_{0}^{1}(\Omega) \times H^{-1}(\Omega)_{\text {weak }}$. For $\bar{\lambda}=0$ the path $\mathcal{C}_{0}$ is bounded in $H_{0}^{1}(\Omega) \times H^{-1}(\Omega)$, with $\lim _{\gamma \rightarrow 0^{+}}\left(y_{\gamma}, \lambda_{\gamma}\right)=(\hat{y}, 0)$ in $H_{0}^{1}(\Omega) \times L^{2}(\Omega)$.

Proof. From $\left(\mathrm{OC}_{\gamma}\right)$ we have for every $\gamma>0$

$$
a\left(y_{\gamma}, y_{\gamma}-y^{*}\right)+\left(\lambda_{\gamma}, y_{\gamma}-y^{*}\right)=\left(f, y_{\gamma}-y^{*}\right) .
$$

Since $\lambda_{\gamma}=\max \left(0, \bar{\lambda}+\gamma\left(y_{\gamma}-\psi\right)\right) \geq 0$ and $\psi-y^{*} \geq 0$ we have

$$
\begin{aligned}
\left(\lambda_{\gamma}, y_{\gamma}-y^{*}\right) & =\left(\lambda_{\gamma}, \frac{\bar{\lambda}}{\gamma}+y_{\gamma}-\psi+\psi-y^{*}-\frac{\bar{\lambda}}{\gamma}\right) \\
& \geq \frac{1}{\gamma}\left(\lambda_{\gamma}, \bar{\lambda}+\gamma\left(y_{\gamma}-\psi\right)\right)-\frac{1}{\gamma}\left(\lambda_{\gamma}, \bar{\lambda}\right) \\
& =\frac{1}{\gamma}\left[\left|\lambda_{\gamma}\right|_{L^{2}}^{2}-\left(\lambda_{\gamma}, \bar{\lambda}\right)\right] .
\end{aligned}
$$

Combined with (13) this implies that

$$
a\left(y_{\gamma}, y_{\gamma}\right)+\frac{1}{\gamma}\left|\lambda_{\gamma}\right|_{L^{2}}^{2} \leq a\left(y_{\gamma}, y^{*}\right)+\left(f, y_{\gamma}-y^{*}\right)+\frac{1}{\gamma}\left(\bar{\lambda}, \lambda_{\gamma}\right)
$$


This estimate, $(6),\left(\mathrm{OC}_{\gamma}\right)$, and the Poincaré-Friedrichs inequality imply that $\mathcal{C}_{r}$ is bounded in $H_{0}^{1}(\Omega) \times H^{-1}(\Omega)$ for every $r>0$. In fact, for $\omega>0$ satisfying $\omega|y|_{H^{1}}^{2} \leq$ $|y|_{H_{0}^{1}}^{2}$, we have

$$
\begin{aligned}
\omega\left|y_{\gamma}\right|_{H^{1}}^{2}+\frac{1}{\gamma}\left|\lambda_{\gamma}\right|_{L^{2}}^{2} \leq & a\left(y_{\gamma}, y_{\gamma}\right)+\frac{1}{\gamma}\left|\lambda_{\gamma}\right|_{L^{2}}^{2} \\
\leq & \mu\left|y_{\gamma}\right|_{H^{1}}\left|y^{*}\right|_{H^{1}}+|f|_{H^{-1}}\left(\left|y_{\gamma}\right|_{H^{1}}+\left|y^{*}\right|_{H^{1}}\right)+\frac{1}{\gamma}|\bar{\lambda}|_{L^{2}}\left|\lambda_{\gamma}\right|_{L^{2}} \\
\leq & \frac{\omega}{4}\left|y_{\gamma}\right|_{H^{1}}^{2}+\frac{\mu^{2}}{\omega}\left|y^{*}\right|_{H^{1}}^{2}+\frac{\omega}{2}\left|y_{\gamma}\right|_{H^{1}}^{2}+\frac{1}{2 \omega}|f|_{H^{-1}}^{2} \\
& +\frac{1}{2 \gamma}\left|\lambda_{\gamma}\right|_{L^{2}}^{2}+\frac{1}{2 \gamma}|\bar{\lambda}|_{L^{2}}^{2}+|f|_{H^{-1}}\left|y^{*}\right|_{H^{1}}
\end{aligned}
$$

and hence

$$
\frac{\omega}{4}\left|y_{\gamma}\right|_{H^{1}}^{2}+\frac{1}{2 \gamma}\left|\lambda_{\gamma}\right|_{L^{2}}^{2} \leq \frac{\mu^{2}}{\omega}\left|y^{*}\right|_{H^{1}}^{2}+\frac{1}{2 \omega}|f|_{H^{-1}}+|f|_{H^{-1}}\left|y^{*}\right|_{H^{1}}+\frac{1}{2 \gamma}|\bar{\lambda}|_{L^{2}}^{2} .
$$

This estimate implies that $\left\{y_{\gamma}: \gamma \geq r\right\}$ is bounded in $H_{0}^{1}(\Omega)$ for every $r>0$. The first equation of $\left(\mathrm{OC}_{\gamma}\right)$ implies that $\left\{\lambda_{\gamma}: \gamma \geq r\right\}$ is bounded in $H^{-1}(\Omega)$ as well. From Proposition 2.1 we have that $\lim _{\gamma \rightarrow \infty}\left(y_{\gamma}, \lambda_{\gamma}\right)=\left(y^{*}, \lambda^{*}\right)$ in $H_{0}^{1}(\Omega) \times H^{-1}(\Omega)_{\text {weak }}$. If $\bar{\lambda}=0$, then from (14), (6), and $\left(\mathrm{OC}_{\gamma}\right)$ the path $\mathcal{C}_{o}$ is bounded in $H_{0}^{1}(\Omega) \times H^{-1}(\Omega)$ and $\lambda_{\gamma} \rightarrow 0$ in $L^{2}(\Omega)$ for $\gamma \rightarrow 0^{+}$. From $\left(\mathrm{OC}_{\gamma}\right)$ and the optimality condition for $(\hat{\mathrm{P}})$ we have

$$
a\left(y_{\gamma}-\hat{y}, y_{\gamma}-\hat{y}\right)+\left(\lambda_{\gamma}, y_{\gamma}-\hat{y}\right)=0
$$

and hence $\lim _{\gamma \rightarrow 0^{+}} y_{\gamma}=\hat{y}$ in $H_{0}^{1}(\Omega)$.

Proposition 3.3. The path $\mathcal{C}_{r}$ is globally Lipschitz in $H_{0}^{1}(\Omega) \times H^{-1}(\Omega)$ for every $r>0$. If $\bar{\lambda}=0$, then $\mathcal{C}_{0}$ is globally Lipschitz continuous.

Proof. Let $\gamma, \bar{\gamma} \in[r, \infty)$ be arbitrary. Then

$$
A\left(y_{\gamma}-y_{\bar{\gamma}}\right)+\left(\bar{\lambda}+\gamma\left(y_{\gamma}-\psi\right)\right)^{+}-\left(\bar{\lambda}+\bar{\gamma}\left(y_{\bar{\gamma}}-\psi\right)\right)^{+}=0 .
$$

Taking the inner-product with $y_{\gamma}-y_{\bar{\gamma}}$ and using the monotonicity and Lipschitz continuity (with constant $L=1$ ) of $x \mapsto \max (0, x)$, we find

$$
\begin{aligned}
a\left(y_{\gamma}-y_{\bar{\gamma}},\right. & \left.y_{\gamma}-y_{\bar{\gamma}}\right) \\
& \leq\left|\left(\left(\bar{\lambda}+\gamma\left(y_{\gamma}-\psi\right)\right)^{+}-\left(\bar{\lambda}+\bar{\gamma}\left(y_{\bar{\gamma}}-\psi\right)\right)^{+}, y_{\gamma}-y_{\bar{\gamma}}\right)\right| \\
& \leq|\gamma-\bar{\gamma}|\left|y_{\gamma}-\psi\right|_{L^{2}}\left|y_{\gamma}-y_{\bar{\gamma}}\right|_{L^{2}} .
\end{aligned}
$$

By Lemma 3.2 the set $\left\{y_{\gamma}\right\}_{\gamma \geq r}$ is bounded in $H_{0}^{1}(\Omega)$. Hence there exists $K_{1}>0$ such that

$$
\nu\left|y_{\gamma}-y_{\bar{\gamma}}\right|_{H_{0}^{1}}^{2} \leq K_{1}|\gamma-\bar{\gamma}| \cdot\left|y_{\gamma}-y_{\bar{\gamma}}\right|_{L^{2}}
$$

and by Poincaré's inequality there exists $K_{2}>0$ such that

$$
\left|y_{\gamma}-y_{\bar{\gamma}}\right|_{H_{0}^{1}} \leq K_{2}|\gamma-\bar{\gamma}| \quad \text { for all } \quad \gamma \geq r, \bar{\gamma} \geq r .
$$


Let us recall here that $|y|_{H_{0}^{1}}=|\nabla y|_{L^{2}}$. Lipschitz continuity of $\gamma \mapsto \lambda_{\gamma}$ from $[r, \infty)$ to $H^{-1}(\Omega)$ follows from the first equation in $\left(\mathrm{OC}_{\gamma}\right)$. For $\bar{\lambda}=0$ the set $\left\{y_{\gamma}\right\}_{\gamma \geq 0}$ is bounded in $H_{0}^{1}(\Omega)$. The remainder of the proof remains identical.

Lemma 3.4. For every subset $I \subset[r, \infty), r>0$, the mapping $\gamma \mapsto \lambda_{\gamma}$ is globally Lipschitz from $I$ to $L^{2}(\Omega)$.

Proof. For $0<\gamma_{1} \leq \gamma_{2}$ we have by $\left(\mathrm{OC}_{\gamma}\right)$

$$
\begin{aligned}
\left|\lambda_{\gamma_{1}}-\lambda_{\gamma_{2}}\right|_{L^{2}} & =\left|\left(\bar{\lambda}+\gamma_{1}\left(y_{\gamma_{1}}-\psi\right)\right)^{+}-\left(\bar{\lambda}+\gamma_{2}\left(y_{\gamma_{2}}-\psi\right)\right)^{+}\right|_{L^{2}} \\
& \leq\left(K_{3} \gamma_{1}+K_{1}+|\psi|_{L^{2}}\right)\left|\gamma_{1}-\gamma_{2}\right|
\end{aligned}
$$

for some constant $K_{3}>0$.

We shall use the following notation:

$$
S_{\gamma}=\left\{x \in \Omega: \bar{\lambda}(x)+\gamma\left(y_{\gamma}-\psi\right)(x)>0\right\} .
$$

Further we set

$$
g(\gamma)=\bar{\lambda}+\gamma\left(y_{\gamma}-\psi\right)
$$

Since $\gamma \mapsto y_{\gamma} \in H_{0}^{1}(\Omega)$ is Lipschitz continuous by Proposition 3.3, there exists a weak accumulation point $\dot{y}\left(=\dot{y}_{\gamma}\right)$ of $\frac{1}{\bar{\gamma}-\gamma}\left(y_{\bar{\gamma}}-y_{\gamma}\right)$ as $\bar{\gamma} \rightarrow \gamma>0$, which is also a strong accumulation point in $L^{2}(\Omega)$. Further $\frac{1}{\bar{\gamma}-\gamma}(g(\bar{\gamma})-g(\gamma))$ has $\dot{g}(\gamma):=y_{\gamma}-\psi+\gamma \dot{y}_{\gamma}$ as a strong accumulation point in $L^{2}(\Omega)$ as $\bar{\gamma} \rightarrow \gamma$. In case $\bar{\gamma}$ approaches $\gamma$ from above (or below), the associated accumulation points $\dot{y}_{\gamma}^{r}$ (or $\dot{y}_{\gamma}^{l}$ ) satisfy certain properties which are described next. In what follows we use $\dot{g}^{r}(\gamma)$ or $\dot{g}^{l}(\gamma)$ whenever $\dot{y}_{\gamma}$ in $\dot{g}(\gamma)$ is replaced by $\dot{y}_{\gamma}^{r}$ and $\dot{y}_{\gamma}^{l}$, respectively.

Proposition 3.5. Let $\gamma>0$, and denote by $\dot{y}_{\gamma}^{r}$ any weak accumulation point of $\frac{1}{\bar{\gamma}-\gamma}\left(y_{\bar{\gamma}}-y_{\gamma}\right)$ in $H_{0}^{1}(\Omega)$ as $\bar{\gamma} \downarrow \gamma$. Set

$$
S_{\gamma}^{+}=S_{\gamma} \cup\left\{x: \bar{\lambda}(x)+\gamma\left(y_{\gamma}(x)-\psi(x)\right)=0 \wedge \dot{g}^{r}(\gamma)(x) \geq 0\right\} .
$$

Then $\dot{y}_{\gamma}^{r}$ satisfies

$$
a\left(\dot{y}_{\gamma}^{r}, v\right)+\left(\left(y_{\gamma}-\psi+\gamma \dot{y}_{\gamma}^{r}\right) \chi_{S_{\gamma}^{+}}, v\right)=0 \text { for all } v \in H_{0}^{1}(\Omega) .
$$

Proof. By $\left(\mathrm{OC}_{\gamma}\right)$ we have for every $v \in H_{0}^{1}(\Omega)$

$$
a\left(y_{\bar{\gamma}}-y_{\gamma}, v\right)+\left(\left(\bar{\lambda}+\bar{\gamma}\left(y_{\bar{\gamma}}-\psi\right)\right)^{+}-\left(\bar{\lambda}+\gamma\left(y_{\gamma}-\psi\right)\right)^{+}, v\right)=0 .
$$

We multiply (17) by $(\bar{\gamma}-\gamma)^{-1}$ and discuss separately the two terms in (17). Clearly, we have

$$
\lim _{\bar{\gamma} \downarrow \gamma}(\bar{\gamma}-\gamma)^{-1} a\left(y_{\bar{\gamma}}-y_{\gamma}, v\right)=a\left(\dot{y}_{\gamma}^{r}, v\right) .
$$

Here and below the limit is taken on the sequence of $\bar{\gamma}$-values, which provides the accumulation point. Lebesgue's bounded convergence theorem allows us to consider the pointwise limits of the integrands. Considering separately the cases $g(\gamma)(x)<0$, $g(\gamma)(x)>0$, and $g(\gamma)(x)=0$, we have

$$
\begin{aligned}
& (\bar{\gamma}-\gamma)^{-1}\left((g(\bar{\gamma}))^{+}-(g(\gamma))^{+}, v\right) \\
& \quad \rightarrow\left(\left(y_{\gamma}-\psi+\gamma \dot{y}_{\gamma}^{r}\right) \chi_{S_{\gamma}^{+}}, v\right) \text { as } \bar{\gamma} \downarrow \gamma
\end{aligned}
$$


which ends the proof.

As a consequence of the proof we obtain the following result.

COROLlaRY 3.6. Let $\gamma>0$, and denote by $\dot{y}_{\gamma}^{l}$ any weak accumulation point of $\frac{1}{\bar{\gamma}-\gamma}\left(y_{\bar{\gamma}}-y_{\gamma}\right)$ in $H_{0}^{1}(\Omega)$ as $\bar{\gamma} \uparrow \gamma$. Set $S_{\gamma}^{-}=S_{\gamma} \cup\left\{x: \bar{\lambda}(x)+\gamma\left(y_{\gamma}(x)-\psi(x)\right)=\right.$ $\left.0 \wedge \dot{g}^{l}(\gamma)(x) \geq 0\right\}$. Then $\dot{y}_{\gamma}^{l}$ satisfies

$$
a\left(\dot{y}_{\gamma}^{l}, v\right)+\left(\left(y_{\gamma}-\psi+\gamma \dot{y}_{\gamma}^{l}\right) \chi_{S_{\gamma}^{-}}, v\right)=0 \quad \text { for all } v \in H_{0}^{1}(\Omega) .
$$

Another corollary of Proposition 3.5 treats the case $\bar{\lambda}=0$.

COROLlary 3.7. Let $\bar{\lambda}=0$, and assume that (11) holds. Then the right- and left- derivatives $\dot{y}_{\gamma}^{r}$ and $\dot{y}_{\gamma}^{l}$ of $\gamma \mapsto y_{\gamma}, \gamma \in(0, \infty)$, exist and are given by

$$
\begin{array}{rr}
a\left(\dot{y}_{\gamma}^{r}, v\right)+\left(\left(y_{\gamma}-\psi+\gamma \dot{y}_{\gamma}^{r}\right) \chi_{\left\{y_{\gamma}>\psi\right\}}, v\right)=0 & \text { for all } v \in H_{0}^{1}(\Omega), \\
a\left(\dot{y}_{\gamma}^{l}, v\right)+\left(\left(y_{\gamma}-\psi+\gamma \dot{y}_{\gamma}^{l}\right) \chi_{\left\{y_{\gamma} \geq \psi\right\}}, v\right)=0 & \text { for all } v \in H_{0}^{1}(\Omega) .
\end{array}
$$

Proof. Let $\bar{\gamma} \downarrow \gamma$. By Proposition 2.2 any accumulation point $\dot{y}_{\gamma}^{r}$ of $(\bar{\gamma}-\gamma)^{-1}\left(y_{\bar{\gamma}}-\right.$ $\left.y_{\gamma}\right)$ satisfies $\dot{y}_{\gamma}^{r} \leq 0$ and hence

$$
S_{\gamma}^{+}=\left\{x \in \Omega: y_{\gamma}(x)>\psi(x)\right\} \cup\left\{x \in \Omega: y_{\gamma}(x)=\psi(x) \wedge \dot{y}_{\gamma}^{r}(x)=0\right\} .
$$

Observe that

$$
\left(y_{\gamma}-\psi+\gamma \dot{y}_{\gamma}^{r}\right) \chi_{S_{\gamma}^{+}}=\left(y_{\gamma}-\psi+\gamma \dot{y}_{\gamma}^{r}\right) \chi_{\left\{y_{\gamma}>\psi\right\}} .
$$

This implies that every accumulation point $\dot{y}_{\gamma}^{r}$ satisfies (20). Since the solution to (20) is unique, the directional derivative from the right exists.

Similarly, if $\bar{\gamma} \uparrow \gamma$, by Proposition 2.2 we have $S_{\gamma}^{-}=\left\{x \in \Omega: y_{\gamma}(x) \geq \psi(x)\right\}$, and (21) follows.

Henceforth we set

$$
S_{\gamma}^{\circ}=\left\{x \in \Omega: \bar{\lambda}(x)+\gamma\left(y_{\gamma}-\psi\right)(x)=0\right\} .
$$

Corollary 3.8. If meas $\left(S_{\gamma}^{\circ}\right)=0$, then $\gamma \mapsto y_{\gamma} \in H_{0}^{1}(\Omega)$ is differentiable at $\gamma$, and the derivative $\dot{y}_{\gamma}$ satisfies

$$
a\left(\dot{y}_{\gamma}, v\right)+\left(\left(y_{\gamma}-\psi+\gamma \dot{y}_{\gamma}\right) \chi_{S_{\gamma}}, v\right)=0 \quad \text { for all } v \in H_{0}^{1}(\Omega) .
$$

Proof. Let $z$ denote the difference of two accumulation points of $(\bar{\gamma}-\gamma)^{-1}\left(y_{\bar{\gamma}}-\right.$ $\left.y_{\gamma}\right)$ as $\bar{\gamma} \rightarrow \gamma$. As a consequence of (16) and (19)

$$
a(z, v)+\gamma\left(z \chi_{S_{\gamma}}, v\right)=0 \quad \text { for all } v \in H_{0}^{1}(\Omega) .
$$

This implies that $z=0$ by (6). Consequently, accumulation points are unique, and by (16), (19) they satisfy (22).

The assumption meas $\left(S_{\gamma}^{o}\right)=0$ in Corollary 3.8 reflects the lack of differentiability of the max-operation in $\left(\mathrm{OC}_{\gamma}\right)$.

4. The primal-dual path value functional. In this section we investigate the value function associated with $\left(\mathrm{P}_{\gamma}\right)$ and study its monotonicity and smoothness properties.

Definition 4.1. The functional

$$
\gamma \mapsto V(\gamma)=J\left(y_{\gamma} ; \gamma\right)=\frac{1}{2} a\left(y_{\gamma}, y_{\gamma}\right)-\left(f, y_{\gamma}\right)+\frac{1}{2 \gamma}\left|\left(\bar{\lambda}+\gamma\left(y_{\gamma}-\psi\right)\right)^{+}\right|_{L^{2}}^{2}
$$


defined on $(0, \infty)$ is called the primal-dual path value functional.

Let us start by studying first order differentiability properties of $V$.

Proposition 4.2. The value function $V$ is differentiable with

$$
\dot{V}(\gamma)=-\frac{1}{2 \gamma^{2}} \int_{\Omega}\left|\left(\bar{\lambda}+\gamma\left(y_{\gamma}-\psi\right)\right)^{+}\right|^{2}+\frac{1}{\gamma} \int_{\Omega}\left(\bar{\lambda}+\gamma\left(y_{\gamma}-\psi\right)\right)^{+}\left(y_{\gamma}-\psi\right) .
$$

Corollary 4.3. For $\bar{\lambda}=0$ we have $\dot{V}(\gamma)=\frac{1}{2} \int_{\Omega}\left|\left(y_{\gamma}-\psi\right)^{+}\right|^{2} \geq 0$ and $\dot{V}(\gamma)>0$ unless $y_{\gamma}$ is feasible. For $\bar{\lambda}$ satisfying (12) and with (11) holding, we have $y_{\gamma} \leq \psi$ and hence $\dot{V}(\gamma) \leq 0$ for $\gamma \in(0, \infty)$.

In either of the two cases $\dot{V}(\gamma)=0$ implies that $y_{\gamma}$ solves $(\hat{\mathrm{P}})$.

Proof. We show only that $\dot{V}(\gamma)=0$ implies that $y_{\gamma}$ solves $(\hat{\mathrm{P}})$. The rest of the assertion follows immediately from Proposition 4.2.

If $\bar{\lambda}=0$, then $\dot{V}(\gamma)=0$ yields $y_{\gamma} \leq \psi$. Thus, $\lambda_{\gamma}=0$, and hence $y_{\gamma}$ solves $(\hat{\mathrm{P}})$.

If (11) and (12) are satisfied, then $y_{\gamma} \leq \psi$ and $\dot{V}(\gamma)=0$ implies $\gamma\left(y_{\gamma}-\psi\right) \leq$ $\bar{\lambda}+\gamma\left(y_{\gamma}-\psi\right) \leq 0$. As a consequence $\lambda_{\gamma}=0$, and $y_{\gamma}$ solves $(\hat{\mathrm{P}})$.

Proof of Proposition 4.2. For $\bar{\gamma}, \gamma \in(0, \infty)$ we find

$$
\begin{aligned}
& \frac{1}{2} a\left(y_{\bar{\gamma}}+y_{\gamma}, y_{\bar{\gamma}}-y_{\gamma}\right)-\left(f, y_{\bar{\gamma}}-y_{\gamma}\right) \\
& \quad+\frac{1}{2}\left(\left(\bar{\lambda}+\bar{\gamma}\left(y_{\bar{\gamma}}-\psi\right)\right)^{+}+\left(\bar{\lambda}+\gamma\left(y_{\gamma}-\psi\right)\right)^{+}, y_{\bar{\gamma}}-y_{\gamma}\right)=0,
\end{aligned}
$$

and consequently

$$
\begin{aligned}
V(\bar{\gamma})-V(\gamma)= & \frac{1}{2} a\left(y_{\bar{\gamma}}, y_{\bar{\gamma}}\right)-\frac{1}{2} a\left(y_{\gamma}, y_{\gamma}\right)-\left(f, y_{\bar{\gamma}}-y_{\gamma}\right) \\
& +\frac{1}{2 \bar{\gamma}} \int_{\Omega}\left|\left(\bar{\lambda}+\bar{\gamma}\left(y_{\bar{\gamma}}-\psi\right)\right)^{+}\right|^{2}-\frac{1}{2 \gamma} \int_{\Omega}\left|\left(\bar{\lambda}+\gamma\left(y_{\gamma}-\psi\right)\right)^{+}\right|^{2} \\
= & \frac{1}{2 \bar{\gamma}} \int_{\Omega}\left|\left(\bar{\lambda}+\bar{\gamma}\left(y_{\bar{\gamma}}-\psi\right)\right)^{+}\right|^{2}+\frac{1}{2 \gamma} \int_{\Omega}-\left|\left(\bar{\lambda}+\gamma\left(y_{\gamma}-\psi\right)\right)^{+}\right|^{2} \\
& \quad+\frac{1}{2} \int_{\Omega}-\left(\left(\bar{\lambda}+\bar{\gamma}\left(y_{\bar{\gamma}}-\psi\right)\right)^{+}+\left(\bar{\lambda}+\gamma\left(y_{\gamma}-\psi\right)\right)^{+}\right)\left(y_{\bar{\gamma}}-y_{\gamma}\right) \\
= & \int_{P_{\bar{\gamma}} \cap P_{\gamma}} z+\int_{P_{\bar{\gamma}} \cap N_{\gamma}} z+\int_{P_{\gamma} \cap N_{\bar{\gamma}}} z=\mathcal{I}_{1}+\mathcal{I}_{2}+\mathcal{I}_{3},
\end{aligned}
$$

where $z$ stands for the sum of the kernels on the left of the above equalities,

$$
P_{\gamma}=\left\{x: \bar{\lambda}+\gamma\left(y_{\gamma}-\psi\right)>0\right\}, \quad N_{\gamma}=\left\{x: \bar{\lambda}+\gamma\left(y_{\gamma}-\psi\right)<0\right\},
$$

and $P_{\bar{\gamma}}, N_{\bar{\gamma}}$ are defined analogously. For $\mathcal{I}_{2}$ we have

$$
\begin{aligned}
& \left|\mathcal{I}_{2}\right| \leq \frac{1}{2} \int_{P_{\bar{\gamma}} \cap N_{\gamma}} \frac{1}{\bar{\gamma}}\left(\bar{\lambda}+\bar{\gamma}\left(y_{\bar{\gamma}}-\psi\right)\right)^{2}+\left|\bar{\lambda}+\bar{\gamma}\left(y_{\bar{\gamma}}-\psi\right)\right|\left|y_{\bar{\gamma}}-y_{\gamma}\right| \\
& \leq \frac{1}{2} \int_{\Omega} \frac{1}{\bar{\gamma}}\left(\bar{\gamma}\left(y_{\bar{\gamma}}-\psi\right)-\gamma\left(y_{\gamma}-\psi\right)\right)^{2}+\left|y_{\bar{\gamma}}-y_{\gamma}\right|\left(\left|\bar{\gamma} y_{\bar{\gamma}}-\gamma y_{\gamma}\right|+|\bar{\gamma}-\gamma||\psi|\right),
\end{aligned}
$$

and hence by Proposition 3.3

$$
\lim _{\bar{\gamma} \rightarrow \gamma} \frac{1}{\bar{\gamma}-\gamma} \quad\left|\mathcal{I}_{2}\right|=0
$$


Analogously one verifies that

$$
\lim _{\bar{\gamma} \rightarrow \gamma} \frac{1}{\bar{\gamma}-\gamma} \quad\left|\mathcal{I}_{3}\right|=0
$$

On $P_{\bar{\gamma}} \cap P_{\gamma}$ we have

$$
\begin{aligned}
z=\frac{1}{2 \bar{\gamma}}( & \left.\bar{\lambda}+\bar{\gamma}\left(y_{\bar{\gamma}}-\psi\right)\right)^{2} \\
& \quad-\frac{1}{2 \gamma}\left(\bar{\lambda}+\gamma\left(y_{\gamma}-\psi\right)\right)^{2}-\frac{1}{2}\left(2 \bar{\lambda}+\bar{\gamma}\left(y_{\bar{\gamma}}-\psi\right)+\gamma\left(y_{\gamma}-\psi\right)\right)\left(y_{\bar{\gamma}}-y_{\gamma}\right) \\
=\frac{\gamma-\bar{\gamma}}{2 \bar{\gamma} \gamma}\left(\bar{\lambda}+\bar{\gamma}\left(y_{\bar{\gamma}}-\psi\right)\right)^{2} & \\
& +\frac{1}{2 \gamma}\left[2 \bar{\lambda}\left(\bar{\gamma}\left(y_{\bar{\gamma}}-\psi\right)-\gamma\left(y_{\gamma}-\psi\right)\right)+\bar{\gamma}^{2}\left(y_{\bar{\gamma}}-\psi\right)^{2}-\gamma^{2}\left(y_{\gamma}-\psi\right)^{2}\right] \\
& \quad-\frac{1}{2}\left(2 \bar{\lambda}+\bar{\gamma}\left(y_{\bar{\gamma}}-\psi\right)+\gamma\left(y_{\gamma}-\psi\right)\right)\left(y_{\bar{\gamma}}-y_{\gamma}\right) \\
=\frac{\gamma-\bar{\gamma}}{2 \bar{\gamma} \gamma} & \left(\bar{\lambda}+\bar{\gamma}\left(y_{\bar{\gamma}}-\psi\right)\right)^{2}+\frac{\bar{\lambda}}{\gamma}\left[\bar{\gamma}\left(y_{\bar{\gamma}}-\psi\right)-\gamma\left(y_{\bar{\gamma}}-\psi\right)\right] \\
& +\frac{1}{2}\left[\frac{\bar{\gamma}^{2}}{\gamma}\left(y_{\bar{\gamma}}-\psi\right)^{2}-\bar{\gamma}\left(y_{\bar{\gamma}}-\psi\right)^{2}+(\bar{\gamma}-\gamma)\left(y_{\bar{\gamma}}-\psi\right)\left(y_{\gamma}-\psi\right)\right],
\end{aligned}
$$

and thus on $P_{\bar{\gamma}} \cap P_{\bar{\gamma}}$

$$
\begin{aligned}
(\bar{\gamma}-\gamma)^{-1} z & =\frac{-1}{2 \bar{\gamma} \gamma}\left(\bar{\lambda}+\bar{\gamma}\left(y_{\gamma}-\psi\right)\right)^{2}+\frac{\bar{\lambda}}{\gamma}\left(y_{\bar{\gamma}}-\psi\right) \\
+ & \frac{1}{2}\left[\frac{\bar{\gamma}}{\gamma}\left(y_{\bar{\gamma}}-\psi\right)^{2}+\left(y_{\bar{\gamma}}-\psi\right)\left(y_{\gamma}-\psi\right)\right] .
\end{aligned}
$$

By Lebesgue's bounded convergence theorem,

$$
\begin{aligned}
& \lim _{\bar{\gamma} \rightarrow \gamma} \frac{1}{\bar{\gamma}-\gamma} \mathcal{I}_{1}=\lim _{\bar{\gamma} \rightarrow \gamma} \frac{1}{\bar{\gamma}-\gamma} \int_{\Omega} z \chi_{P_{\bar{\gamma} \cap P_{\gamma}}} \\
& =-\frac{1}{2 \gamma^{2}} \int_{\Omega}\left(\left(\bar{\lambda}+\gamma\left(y_{\gamma}-\psi\right)\right)^{+}\right)^{2}+\frac{1}{\gamma} \int_{\Omega}\left(\bar{\lambda}+\gamma\left(y_{\gamma}-\psi\right)\right)^{+}\left(y_{\gamma}-\psi\right) .
\end{aligned}
$$

Together with (24) and (25), this implies the claim.

Remark 4.1. Note that $\dot{V}$ is characterized without recourse to $\dot{y}_{\gamma}$.

The boundedness of $\left\{\gamma^{2} \dot{V}(\gamma)\right\}_{\gamma \geq 0}$ is established next. In what follows we use $(v)^{-}=-\min (0, v)$.

Proposition 4.4. If $\bar{\lambda}=0$ and $a\left(v^{+}, v^{-}\right)=0$ for all $v \in H_{0}^{1}(\Omega)$, then $\left\{\gamma^{2} \dot{V}(\gamma)\right\}_{\gamma \geq 0}$ is bounded. If (11) and (12) hold, then again $\left\{\gamma^{2} \dot{V}(\gamma)\right\}_{\gamma \geq 0}$ is bounded.

Proof. In the case $\bar{\lambda}=0$ we have

$$
a\left(y_{\gamma}-\psi, v\right)+\gamma\left(\left(y_{\gamma}-\psi\right)^{+}, v\right)=(f, v)-a(\psi, v) \quad \text { for all } v \in H_{0}^{1}(\Omega) .
$$

Since $\left(y_{\gamma}-\psi\right) \in H_{0}^{1}(\Omega)$ and $a\left(\left(y_{\gamma}-\psi\right)^{+},\left(y_{\gamma}-\psi\right)^{-}\right)=0$ we have, using (6) with $v=\left(y_{\gamma}-\psi\right)^{+}$,

$$
\nu\left|\left(y_{\gamma}-\psi\right)^{+}\right|_{H_{0}^{1}(\Omega)}^{2}+\gamma\left|\left(y_{\gamma}-\psi\right)^{+}\right|_{L^{2}}^{2} \leq|f|_{L^{2}}\left|\left(y_{\gamma}-\psi\right)^{+}\right|_{H_{0}^{1}}+\mu|\psi|_{H^{1}}\left|y_{\gamma}-\psi\right|_{H^{1}} .
$$


This implies the existence of a constant $K$, depending on $|\psi|_{H^{1}}$ and $|f|_{L^{2}}$ but independent of $\gamma \geq 0$, such that $\gamma\left|\left(y_{\gamma}-\psi\right)^{+}\right|_{L^{2}} \leq K$. Since $\dot{V}(\gamma)=\frac{1}{2} \int_{\Omega}\left|\left(y_{\gamma}-\psi\right)^{+}\right|^{2}$ the claim follows.

Turning to the feasible case with (11) and (12) holding, we have that $y_{\gamma} \leq \psi$ for every $\gamma>0$, and hence $\left(\bar{\lambda}+\gamma\left(y_{\gamma}-\psi\right)\right)(x)>0$ if and only if $\bar{\lambda}(x)>\gamma\left(\psi-y_{\gamma}\right)(x)$. Consequently,

$$
\begin{aligned}
|\dot{V}(\gamma)| & \leq \frac{1}{2 \gamma^{2}} \int_{\Omega}\left|\left(\bar{\lambda}+\gamma\left(y_{\gamma}-\psi\right)\right)^{+}\right|^{2}+\frac{1}{\gamma} \int_{\Omega}\left(\bar{\lambda}+\gamma\left(y_{\gamma}-\psi\right)\right)^{+}\left(\psi-y_{\gamma}\right) \\
& \leq \frac{3}{2 \gamma^{2}}|\bar{\lambda}|_{L^{2}}^{2},
\end{aligned}
$$

which again implies the claim.

Before we investigate $\ddot{V}$, we state a result which connects $\gamma \dot{V}(\gamma),\left|y^{*}-y_{\gamma}\right|_{H_{0}^{1}}$, and $V^{*}-V(\gamma)$, where $V^{*}=\lim _{\gamma \rightarrow \infty} V(\gamma)$. It will be used in section 6.1 for designing a $\gamma$-update strategy.

Proposition 4.5. In the feasible and infeasible cases the following estimate holds true:

$$
\left|y^{*}-y_{\gamma}\right|_{H_{0}^{1}}^{2} \leq \frac{2}{\nu}\left(V^{*}-V(\gamma)-\gamma \dot{V}(\gamma)\right) .
$$

Proof. We have $V^{*}-V(\gamma)=J\left(y^{*}\right)-J\left(y_{\gamma} ; \gamma\right)$ and

$$
\begin{gathered}
J\left(y^{*}\right)-J\left(y_{\gamma} ; \gamma\right) \geq \frac{\nu}{2}\left|y^{*}-y_{\gamma}\right|_{H_{0}^{1}}^{2}+a\left(y_{\gamma}, y^{*}-y_{\gamma}\right)-\left(f, y^{*}-y_{\gamma}\right) \\
-\frac{1}{2 \gamma}\left|\left(\bar{\lambda}+\gamma\left(y_{\gamma}-\psi\right)\right)^{+}\right|_{L^{2}}^{2},
\end{gathered}
$$

where we have used (6). From $\left(\mathrm{OC}_{\gamma}\right)$ we have

$$
a\left(y_{\gamma}, y^{*}-y_{\gamma}\right)-\left(f, y^{*}-y_{\gamma}\right)=-\left(\left(\bar{\lambda}+\gamma\left(y_{\gamma}-\psi\right)\right)^{+}, y^{*}-y_{\gamma}\right)
$$

and hence

$$
\begin{gathered}
J\left(y^{*}\right)-J\left(y_{\gamma} ; \gamma\right) \geq \frac{\nu}{2}\left|y^{*}-y_{\gamma}\right|_{H_{0}^{1}}^{2}-\left(\left(\bar{\lambda}+\gamma\left(y_{\gamma}-\psi\right)\right)^{+}, y^{*}-y_{\gamma}\right) \\
-\frac{1}{2 \gamma}\left|\left(\bar{\lambda}+\gamma\left(y_{\gamma}-\psi\right)\right)^{+}\right|_{L^{2}}^{2} \\
\geq \frac{\nu}{2}\left|y^{*}-y_{\gamma}\right|_{H_{0}^{1}}^{2}-\frac{1}{2 \gamma}\left|\left(\bar{\lambda}+\gamma\left(y_{\gamma}-\psi\right)\right)^{+}\right|_{L^{2}}^{2} \\
+\left(\left(\bar{\lambda}+\gamma\left(y_{\gamma}-\psi\right)\right)^{+}, y_{\gamma}-\psi\right) \\
=\frac{\nu}{2}\left|y^{*}-y_{\gamma}\right|_{H_{0}^{1}}^{2}+\gamma \dot{V}(\gamma) .
\end{gathered}
$$

This completes the proof.

Below we shall assume that $y_{\gamma}-\psi \in C(\bar{\Omega})$. Recall that for dimension $n \leq 3$ and with (6) and (8) holding, we have $y_{\gamma} \in H^{2}(\Omega) \subset C(\bar{\Omega})$.

PROPOSITION 4.6. Let $\dot{y}_{\gamma}$ denote any accumulation point of $(\bar{\gamma}-\gamma)^{-1}\left(y_{\bar{\gamma}}-y_{\gamma}\right)$ as $\bar{\gamma} \rightarrow \gamma$.

(a) If $\bar{\lambda}=0, y_{\gamma}-\psi \in C(\bar{\Omega})$, and (8) is satisfied, then $\gamma \mapsto V(\gamma)$ is twice differentiable at $\gamma$ with

$$
\ddot{V}(\gamma)=\int_{\Omega}\left(y_{\gamma}-\psi\right)^{+} \dot{y}_{\gamma}
$$


(b) For arbitrary $\bar{\lambda}$, if meas $\left(S_{\gamma}^{\circ}\right)=0$, then $\gamma \mapsto V(\gamma)$ is twice differentiable at $\gamma$ with

$$
\begin{aligned}
\ddot{V}(\gamma) & =\frac{1}{\gamma^{3}} \int_{\Omega}\left|\left(\bar{\lambda}+\gamma\left(y_{\gamma}-\psi\right)\right)^{+}\right|^{2} \\
& -\frac{2}{\gamma^{2}} \int_{\Omega}\left(\bar{\lambda}+\gamma\left(y_{\gamma}-\psi\right)\right)^{+}\left(y_{\gamma}-\psi\right) \\
& +\frac{1}{\gamma} \int_{\Omega}\left(y_{\gamma}-\psi\right)\left(y_{\gamma}-\psi+\gamma \dot{y}_{\gamma}\right) \chi_{S_{\gamma}} .
\end{aligned}
$$

Proof. (a) On the subsequence $\gamma_{n}$ realizing the accumulation point, we have that $\lim _{n \rightarrow \infty}\left(\gamma_{n}-\gamma\right)^{-1}\left(\dot{V}\left(\gamma_{n}\right)-\dot{V}(\gamma)\right)$ equals the right-hand side of (26). The claim will be established by verifying that the accumulation points $\dot{y}_{\gamma}$ restricted to $S_{\gamma}=$ $\left\{x: y_{\gamma}(x)-\psi(x)>0\right\}$ are unique. Let $z$ denote the difference of two accumulation points. By (16) and (19) we have

$$
a(z, v)+\gamma(z, v)=0 \text { for all } v \in H_{0}^{1}(\Omega) \text { with } v=0 \text { on } \Omega \backslash S_{\gamma} .
$$

Using (8) and the fact that $S_{\gamma}$ is an open set relative to $\Omega$ due to the continuity of $y_{\gamma}-\psi$, we find that $z=0$ in $S_{\gamma}$, as desired.

(b) Let $\dot{y}_{\gamma}$ denote any accumulation point of $(\bar{\gamma}-\gamma)^{-1}\left(y_{\bar{\gamma}}-y_{\gamma}\right)$ as $\bar{\gamma} \downarrow \gamma$, and recall the notation $g(\gamma)=\bar{\lambda}+\gamma\left(y_{\gamma}-\psi\right)$ and $S_{\gamma}^{+}$from section 3. On the subsequence realizing the accumulation point we find

$$
\begin{aligned}
\lim _{\bar{\gamma} \rightarrow \gamma} & \frac{1}{\bar{\gamma}-\gamma}(\dot{V}(\bar{\gamma})-\dot{V}(\gamma))=\frac{1}{\gamma^{3}} \int_{\Omega}\left|\left(\bar{\lambda}+\gamma\left(y_{\gamma}-\psi\right)\right)^{+}\right|^{2} \\
& -\frac{2}{\gamma^{2}} \int_{\Omega}\left(\bar{\lambda}+\gamma\left(y_{\gamma}-\psi\right)\right)^{+}\left(y_{\gamma}-\psi\right) \\
& +\frac{1}{\gamma} \int_{\Omega}\left(y_{\gamma}-\psi\right)\left(y_{\gamma}-\psi+\gamma \dot{y}_{\gamma}\right) \chi_{S_{\gamma}^{+}} .
\end{aligned}
$$

By assumption, meas $\left(S_{\gamma}^{\circ}\right)=0$ and, hence the right-hand sides of (27) and (28) coincide. Since $\dot{y}_{\gamma}$ is unique by Corollary 3.8 the claim is established.

5. Model functions. In this section we derive low-parameter families of functions which approximate the value functional $V$ and share some of its qualitative properties. We will make use of these models in the numerics section when devising path-following algorithms.

5.1. Infeasible case. Throughout this subsection we assume (8) and

$$
\bar{\lambda}=0, y_{\gamma}-\psi \in C(\bar{\Omega}) \quad \text { for all } \gamma \in(0, \infty) .
$$

Observe that (8), together with the general assumption (6), implies (11). In fact, for any $v \in H_{0}^{1}(\Omega)$ we have $a\left(v, v^{+}\right) \geq \gamma\left|v^{+}\right|^{2}$, and hence $0 \geq a\left(v, v^{+}\right)$implies $v^{+}=0$.

Proposition 5.1. The value function $V$ satisfies $\dot{V}(\gamma) \geq 0$ and $\ddot{V}(\gamma) \leq 0$ for $\gamma \in(0, \infty)$.

Proof. Proposition 4.2 implies that $\dot{V}(\gamma) \geq 0$. Moreover, $y_{\gamma_{2}} \leq y_{\gamma_{1}}$ for $\gamma_{2} \geq \gamma_{1}>0$ and hence $\dot{y}_{\gamma} \leq 0$ a.e. on $S_{\gamma}$. Consequently $\ddot{V}(\gamma) \leq 0$ by Proposition 4.6.

A model function $m$ for the value function $V$ should reflect the sign properties of $V$ and its derivatives. Moreover, $V(0)$ gives the value of $(\hat{\mathrm{P}})$, and hence we shall require that $m(0)=V(0)$. Finally from Lemma 3.2 we conclude that $V$ is bounded on $[0, \infty)$. All these properties are satisfied by functions of the form

$$
m(\gamma)=C_{1}-\frac{C_{2}}{E+\gamma}
$$


with $C_{1} \in \mathbb{R}$. Here $C_{2} \geq 0, E>0$ satisfy

$$
m(0)=V(0)=C_{1}-\frac{C_{2}}{E} .
$$

Other choices for model functions are also conceivable, for example, $\gamma \rightarrow C_{1}-\frac{C_{1}}{(E+\gamma)^{r}}$

with $r>1$. Note, however, that the asymptotic behavior of the model in (30) is such that $\gamma^{2} \dot{m}(\gamma)$ is bounded for $\gamma \rightarrow \infty$. This is consistent with the boundedness of $\gamma^{2} \dot{V}(\gamma)$ for $\gamma \rightarrow \infty$ asserted in Proposition 4.4.

Another reason for choosing (30) is illustrated next. Choosing $v=\left(y_{\gamma}-\psi\right)^{+}$in $\left(\mathrm{OC}_{\gamma}\right)$, we find

$$
a\left(\dot{y}_{\gamma},\left(y_{\gamma}-\psi\right)^{+}\right)+\left|\left(y_{\gamma}-\psi\right)^{+}\right|_{L^{2}}^{2}+\gamma \int_{\Omega}\left(y_{\gamma}-\psi\right)^{+} \dot{y}_{\gamma}=0 .
$$

For the following discussion we

$$
\text { replace } a(\cdot, \cdot) \text { by } E(\cdot, \cdot) \text { with } E>0 \text { a constant, and } V \text { by } m \text {. }
$$

By Proposition 4.2 and (26) the following ordinary differential equation is obtained for $m$ :

$$
(E+\gamma) \ddot{m}(\gamma)+2 \dot{m}(\gamma)=0 .
$$

The solutions to (34) are given by (30). To get an account for the quality of our model in (30) we refer to the left-hand plot of Figure 4 in section 6.

5.2. Feasible case. Throughout this subsection we assume

$$
\text { (11), } \bar{\lambda} \text { satisfies (12), and meas }\left(S_{\gamma}^{\circ}\right)=0 \text { for all } \gamma \in(0, \infty) \text {. }
$$

Proposition 5.2. The value function $V$ satisfies $\dot{V}(\gamma) \leq 0$ and $\ddot{V}(\gamma) \geq 0$ for $\gamma \in(0, \infty)$.

Proof. By Proposition 2.2 we have $y_{\gamma} \leq \psi$ and hence $\dot{V}(\gamma) \leq 0$ by Proposition 4.2. A short computation based on (27) shows that

$$
\ddot{V}(\gamma)=\frac{1}{\gamma^{3}} \int_{\Omega} \chi \bar{\lambda}^{2}+\int_{\Omega} \chi\left(y_{\gamma}-\psi\right) \dot{y}_{\gamma} \geq \frac{1}{\gamma} \int_{\Omega} \chi\left(y_{\gamma}-\psi\right)^{2}+\int_{\Omega} \chi\left(y_{\gamma}-\psi\right) \dot{y}_{\gamma},
$$

where $\chi$ is the characteristic function of the set $S_{\gamma}=\left\{\bar{\lambda}+\gamma\left(y_{\gamma}-\psi\right)>0\right\}$. From (22) we have

$$
\gamma\left|\dot{y}_{\gamma}\right|_{L^{2}\left(S_{\gamma}\right)} \leq\left|\psi-y_{\gamma}\right|_{L^{2}\left(S_{\gamma}\right)}
$$

and hence $\ddot{V}(\gamma) \geq 0$.

An immediate consequence is stated next.

LEMma 5.3. If the solution to the unconstrained problem is not feasible, then $\lim _{\gamma \downarrow 0} V(\gamma)=\infty$.

Proof. Assume that $\lim _{\gamma \downarrow 0} V(\gamma)$ is finite. Then, using $\left(\mathrm{P}_{\gamma}\right)$, there exists a sequence $\gamma_{n} \rightarrow 0$ and $\tilde{y} \in H_{0}^{1}(\Omega)$ such that $y_{\gamma_{n}} \rightarrow \tilde{y}$ weakly in $H_{0}^{1}(\Omega)$, with $y_{\gamma_{n}}$ the solution to $\left(\mathrm{P}_{\gamma_{n}}\right)$, and $\lambda_{\gamma_{n}}=\max \left(0, \bar{\lambda}+\gamma_{n}\left(y_{n}-\psi\right)\right) \rightarrow 0$ in $L^{2}(\Omega)$. Consequently $\tilde{y} \leq \psi$. Taking the limit with respect to $n$ in $\left(\mathrm{OC}_{\gamma_{n}}\right)$, it follows that $\tilde{y} \leq \psi$ is the solution to $(\hat{\mathrm{P}})$, which contradicts our assumption. 
From Lemmas 3.2 and 5.3 and Proposition 5.2 it follows that $\gamma \mapsto V(\gamma), \gamma \in$ $(0, \infty)$, is a monotonically strictly decreasing convex function with $\lim _{\gamma \rightarrow 0^{+}} V(\gamma)=\infty$. All these properties are also satisfied by functions of the form

$$
m(\gamma)=C_{1}-\frac{C_{2}}{E+\gamma}+\frac{B}{\gamma},
$$

provided that $C_{1} \in \mathbb{R}, C_{2} \geq 0, E>0, B>0$, and $C_{2} \leq B$.

We now give the motivation for choosing the model function $m$ for $V$ as in (37). From (22) with $v=\left(y_{\gamma}-\psi\right) \chi$ we get

$$
a\left(\dot{y}_{\gamma},(y-\psi) \chi\right)+\gamma\left(\dot{y}_{\gamma} \chi, y_{\gamma}-\psi\right)+\left(\left(y_{\gamma}-\psi\right) \chi, y_{\gamma}-\psi\right)=0,
$$

where $\chi=\chi_{S_{\gamma}}$. As in the infeasible case we replace $a(\cdot, \cdot)$ by $E(\cdot, \cdot)$, with $E$ a constant, and using (22), we arrive at

$$
(E+\gamma)\left(\dot{y}_{\gamma} \chi, v\right)+\left(\left(y_{\gamma}-\psi\right) \chi, v\right)=0 .
$$

The choice $v=y_{\gamma}-\psi$ implies

$$
(E+\gamma)\left(\dot{y}_{\gamma} \chi, y_{\gamma}-\psi\right)+\left(\left(y_{\gamma}-\psi\right) \chi, y_{\gamma}-\psi\right)=0 .
$$

Note that $\dot{V}(\gamma)$ can be expressed as

$$
\dot{V}(\gamma)=-\frac{1}{2 \gamma^{2}} \int_{\Omega} \bar{\lambda}^{2} \chi+\frac{1}{2} \int_{\Omega}\left(y_{\gamma}-\psi\right)^{2} \chi .
$$

Using (36) and (39) in (38), and replacing $V$ by $m$, due to the substitution for $a(\cdot, \cdot)$, we find

$$
(E+\gamma) \ddot{m}+2 \dot{m}-E \gamma^{-3} \int_{\Omega} \chi \bar{\lambda}^{2}=0 .
$$

We further replace $\int_{\Omega} \chi \bar{\lambda}^{2}$, which is a bounded quantity depending on $\gamma$, by $2 B$, and obtain, as the ordinary differential equation that we propose for the model function $m$ in the feasible case,

$$
(E+\gamma) \ddot{m}+2 \dot{m}-2 \gamma^{-3} E B=0 .
$$

The family of solutions is given by (37). In the right-hand plot of Figure 4 in section 6 we depict the approximation quality of $m(\gamma)$.

6. Path-following algorithms. In this section we study the basic Algorithm B together with a variety of adjustment schemes for the path parameter $\gamma$. For this purpose recall that, depending on the shift parameter $\bar{\lambda}$, the elements $y_{\gamma}$ along the primal-dual path are feasible or infeasible. As we have seen in the previous section, this implies different models for approximating the value function $V$. We will see, however, that for $\gamma>0$ in both cases similar strategies for updating $\gamma$ may be used. When referring to the infeasible or feasible case, (29), respectively (35), is assumed to hold.

The subsequent discussion is based on the following two-dimensional test problems. We point out that the bound $\psi$ in problem P1 below does not satisfy $\psi \in H^{1}(\Omega)$. However, as we shall see, the feasible and infeasible primal-dual path as well as the algorithms introduced subsequently still perform satisfactorily. We include this example since discontinuous obstacles are of practical relevance. 
Test problem $\mathrm{P} 1$. We consider (8) with $a_{i j}=\delta_{i j}$, with $\delta_{i j}$ the Kronecker symbol, $d=0$, and $\Omega=(0,1)^{2}$. We choose

$$
f\left(x_{1}, x_{2}\right)=500 x_{1} \sin \left(5 x_{1}\right) \cos \left(x_{2}\right)
$$

and $\psi \equiv 10$ on $\Omega \backslash K$, and $\psi \equiv 1$ on $K$ with $K=\left\{x \in \Omega: \frac{1}{5} \leq\left\|x-\left(\frac{1}{2}, \frac{1}{2}\right)^{\top}\right\|_{2} \leq \frac{2}{5}\right\}$. The solution $y^{*}$, the obstacle $\psi$, and the active set $\mathcal{A}^{*}$ at the solution are shown in Figure 1.
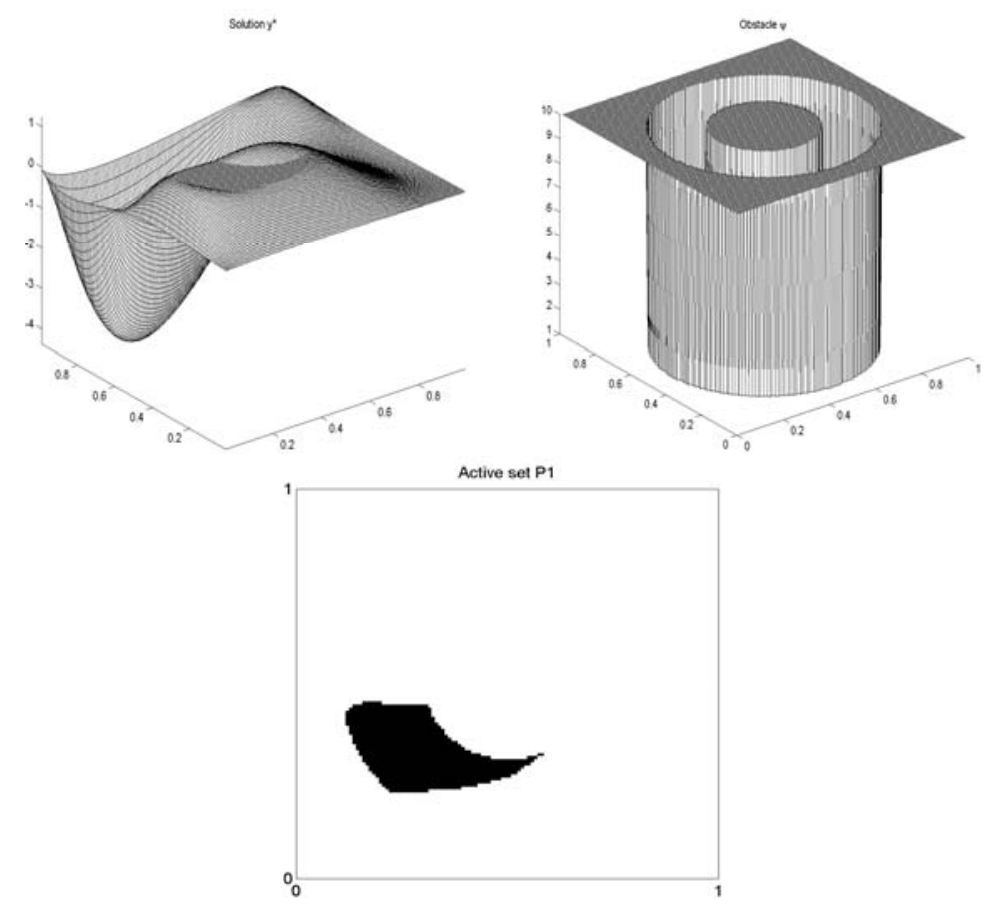

FIG. 1. Optimal solution $y^{*}$ (upper left plot), obstacle $\psi$ (upper right plot), and the active set $\mathcal{A}^{*}$ (lower plot) for test problem $\mathrm{P} 1$.

Test problem P2. Again we consider (8), with $a_{i j}, d$, and $\Omega$ as before, and define

$$
y^{\dagger}:= \begin{cases}x_{1} & \text { on } T_{1}:=\left\{x \in \Omega: x_{2} \leq x_{1} \wedge x_{2} \leq 1-x_{1}\right\}, \\ 1-x_{2} & \text { on } T_{2}:=\left\{x \in \Omega: x_{2} \leq x_{1} \wedge x_{2} \geq 1-x_{1}\right\}, \\ 1-x_{1} & \text { on } T_{3}:=\left\{x \in \Omega: x_{2} \geq x_{1} \wedge x_{2} \geq 1-x_{1}\right\}, \\ x_{2} & \text { on } T_{4}:=\left\{x \in \Omega: x_{2} \geq x_{1} \wedge x_{2} \leq 1-x_{1}\right\} .\end{cases}
$$

The obstacle $\psi$ is defined by $\psi \equiv y^{\dagger}$ on $S_{1}:=\left\{x \in \Omega:\left\|x-\left(\frac{1}{2}, \frac{1}{2}\right)^{\top}\right\|_{\infty} \leq \frac{1}{4}\right\}, \psi \equiv \frac{1}{4}$ on $S_{2} \backslash S_{1}$, and

$$
\psi:= \begin{cases}2 x_{1} & \text { on } T_{1} \cap\left(\Omega \backslash S_{2}\right), \\ \frac{1}{4}-2\left(x_{2}-\frac{7}{8}\right) & \text { on } T_{2} \cap\left(\Omega \backslash S_{2}\right), \\ \frac{1}{4}-2\left(x_{1}-\frac{7}{8}\right) & \text { on } T_{3} \cap\left(\Omega \backslash S_{2}\right), \\ 2 x_{2} & \text { on } T_{4} \cap\left(\Omega \backslash S_{2}\right),\end{cases}
$$

with $S_{2}:=\left\{x \in \Omega:\left\|x-\left(\frac{1}{2}, \frac{1}{2}\right)^{\top}\right\|_{\infty} \leq \frac{3}{8}\right\}$. The forcing term is given by

$$
(f, \phi)_{L^{2}}=\int_{\Omega^{+}} \phi(s) d s+\left(\chi_{S_{1}}, \phi\right)_{L^{2}}+\int_{S_{1} \cap \Omega^{+}} \phi(s) d s \quad \text { for all } \phi \in H_{0}^{1}(\Omega),
$$


where $\Omega^{+}:=\left\{x \in \Omega: x_{2}=x_{1}\right\} \cup\left\{x \in \Omega: x_{2}=1-x_{1}\right\}$. We recall that for $\phi \in H_{0}^{1}(\Omega)$, $\Omega \subset \mathbb{R}^{2}$, the traces along smooth curves are well defined. The solution $y^{*}$ is given by $y^{*}=y^{\dagger}$. The active or coincidence set at the solution is $\mathcal{A}^{*}=S_{1}$. The Lagrange multiplier $\lambda^{*}=f+\Delta y^{*}$ is in $H^{-1}(\Omega)$ and enjoys no extra regularity. In Figure 2 we display the optimal solution $y^{*}$, the obstacle $\psi$, and the active set $\mathcal{A}^{*}$.

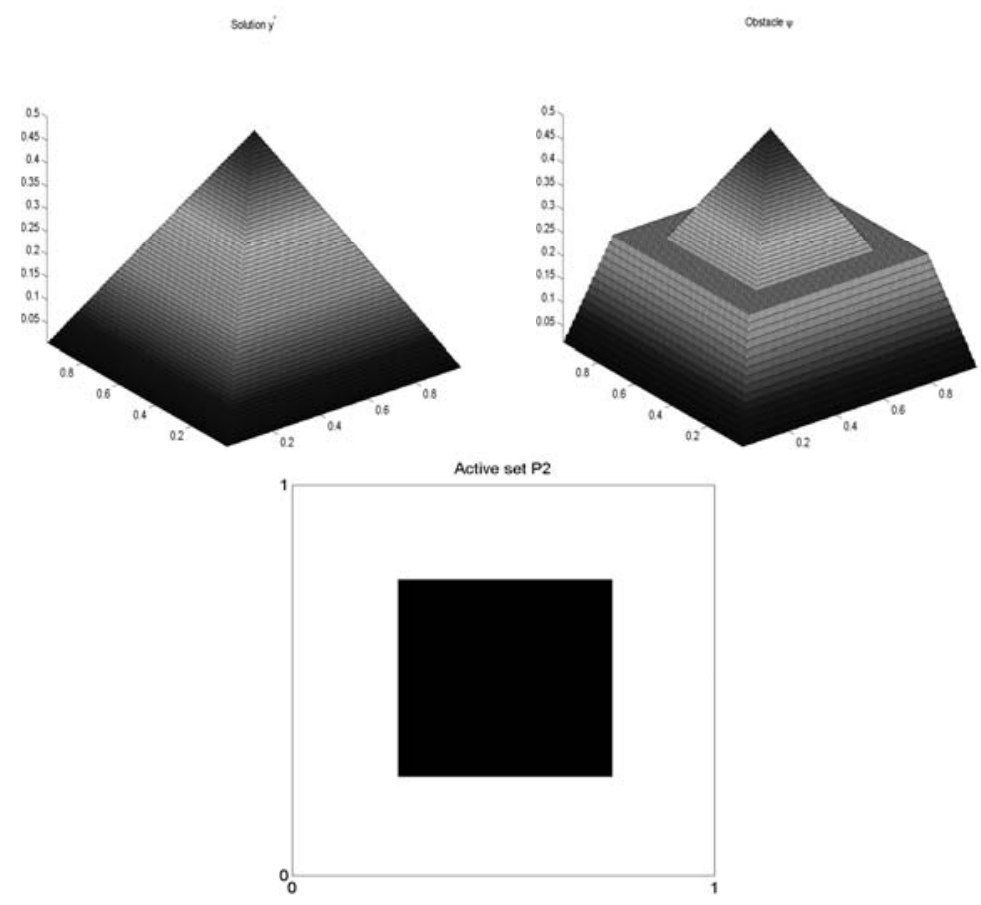

FIG. 2. Optimal solution $y^{*}$ (upper left plot), obstacle $\psi$ (upper right plot), and the active set $\mathcal{A}^{*}$ (lower plot) for test problem $\mathrm{P} 2$.

Test problem P3. For this test problem (8) is satisfied. We therefore obtain $y^{*} \in H^{2}(\Omega)$ and $\lambda^{*} \in L^{2}(\Omega)$. The coefficients $a_{i j}$ and $d$ as well as $\Omega$ are as before. The volume force $f$ is given by $f=-\Delta v$ with $v\left(x_{1}, x_{2}\right)=\sin \left(3 \pi x_{1}\right) \sin \left(3 \pi x_{2}\right)$. Further, we have $\psi=\frac{1}{4}-\frac{1}{10} \sin \left(\pi x_{1}\right) \sin \left(\pi x_{2}\right)$. The optimal solution $y^{*}$, the Lagrange multiplier $\lambda^{*}$, and the active set at $y^{*}$ are displayed in Figure 3 .

Unless specified otherwise, the subsequent algorithms are initialized by $y_{0}=$ $(-\Delta)^{-1} f$, where $-\Delta$ denotes the Laplacian with homogeneous Dirichlet boundary conditions. The initial Lagrange multiplier is chosen as $\lambda_{0}=\gamma_{0} \chi_{\left\{y_{0}>\psi\right\}}\left(y_{0}-\psi\right)$.

The discretization of $-\Delta$ is based on the classical five-point finite difference stencil. We denote the mesh size by $h$, which we occasionally drop for convenience. The forcing term $f$ in $\mathrm{P} 2$ is discretized by $f=-\Delta y^{\dagger}+\chi_{S_{1}} e+\chi_{S_{1}}\left(-\Delta y^{\dagger}\right)$, where $e$ is the vector of all ones and $\chi_{S_{1}}$ represents a diagonal matrix with entry $\left(\chi_{S_{1}}\right)_{i i}=1$ for grid points $x_{i} \in S_{1}$ and $\left(\chi_{S_{1}}\right)_{i i}=0$ otherwise. Above $y^{\dagger}$ denotes the grid function corresponding to (41).

6.1. A strategy based on model functions - exact path-following. As outlined in section 5, there are good reasons to trust our model functions (30) and (37) in the infeasible and feasible cases, respectively. Let us start by focusing on the infeasible case. The model is given by $m(\gamma)=C_{1}-C_{2}(E+\gamma)^{-1}$. For determining 

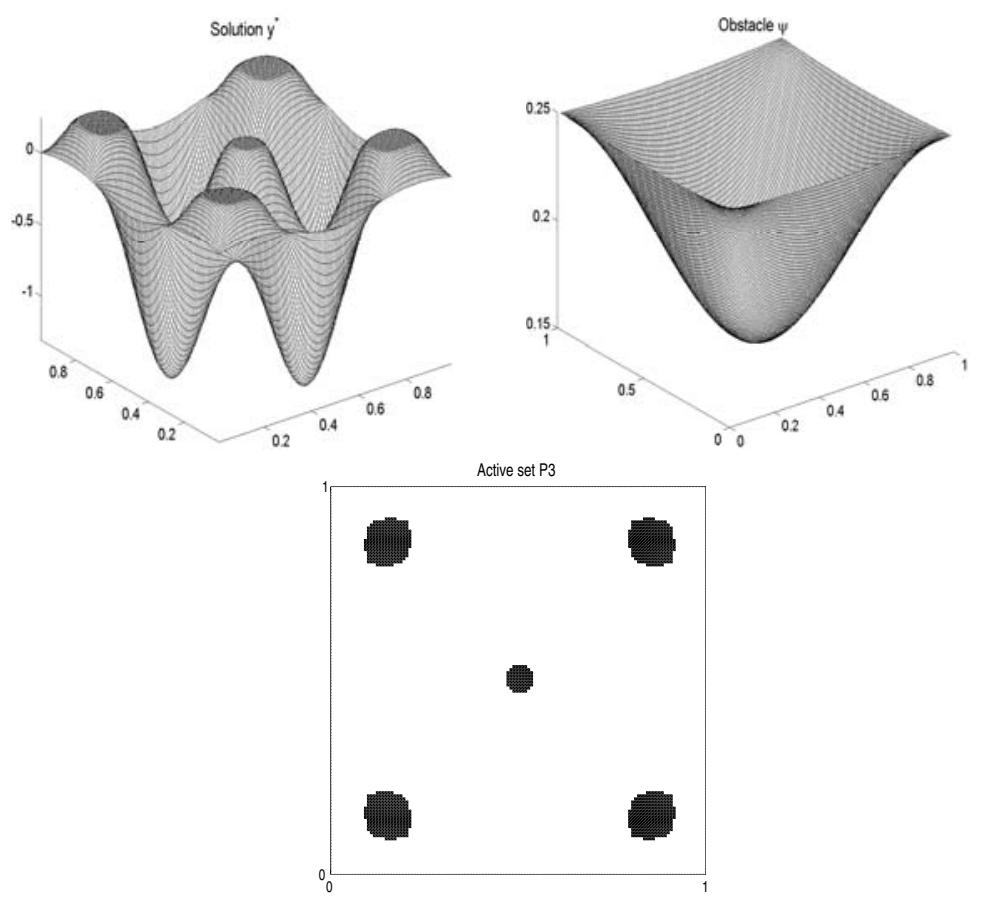

FIG. 3. Optimal solution $y^{*}$ (upper left plot), obstacle $\psi$ (upper right plot), and the active set $\mathcal{A}^{*}$ (lower plot) for test problem $\mathrm{P} 3$.

the three parameters $C_{1}, C_{2}$, and $E$, we use the information $V(0), V(\gamma), \dot{V}(\gamma)$, which, by Proposition 4.2, is available from one solve of the unconstrained problem $(\hat{\mathrm{P}})$ and one solve for $(\mathrm{P} \gamma)$. The conditions

$$
m(0)=V(0), \quad m(\gamma)=V(\gamma), \quad \dot{m}(\gamma)=\dot{V}(\gamma)
$$

yield

$$
\begin{aligned}
E & =\gamma^{2} \dot{V}(\gamma)(V(\gamma)-V(0)-\gamma \dot{V}(\gamma))^{-1}, \\
C_{2} & =\gamma^{-1} E(E+\gamma)(V(\gamma)-V(0)), \\
C_{1} & =V(0)+C_{2} E^{-1} .
\end{aligned}
$$

We could have used an alternative reference value $\gamma_{r} \in(0, \gamma)$ and computed $m\left(\gamma_{r}\right)=$ $V\left(\gamma_{r}\right)$ instead of $m(0)=V(0)$. In Figure 4 we compare $V(\gamma)$ to $m(\gamma)$ for different values of the coefficients $\left(C_{1}, C_{2}, E\right)$. These coefficients depend on different values $y_{f}$ for $\gamma$ (in (42)) produced by Algorithm EP (see below) for problem P1. The solid line corresponds to $V(\gamma)$. The corresponding $\gamma$-values $\gamma_{f}$ for (42) are depicted in the legend of the left plot in Figure 4. The dotted and dashed line belong to rather small $\gamma$-values, and the dashed-dotted and the circled lines to large $\gamma_{f}$ in (42). As we can see, the dotted line is accurate in the range of relatively small $\gamma_{f}$, while the other lines are more accurate for large $\gamma_{f}$. From now on we consider only the choices $\gamma_{r}=0$ and $\gamma=\gamma_{k}$ in (42) when updating $\gamma_{k}$.

Next we discuss properties of the model parameters $E, C_{1}, C_{2}$ according to (43). For this purpose assume that the solution $\hat{y}$ to $(\hat{\mathrm{P}})$ is not feasible for $(\mathrm{P})$. Then 

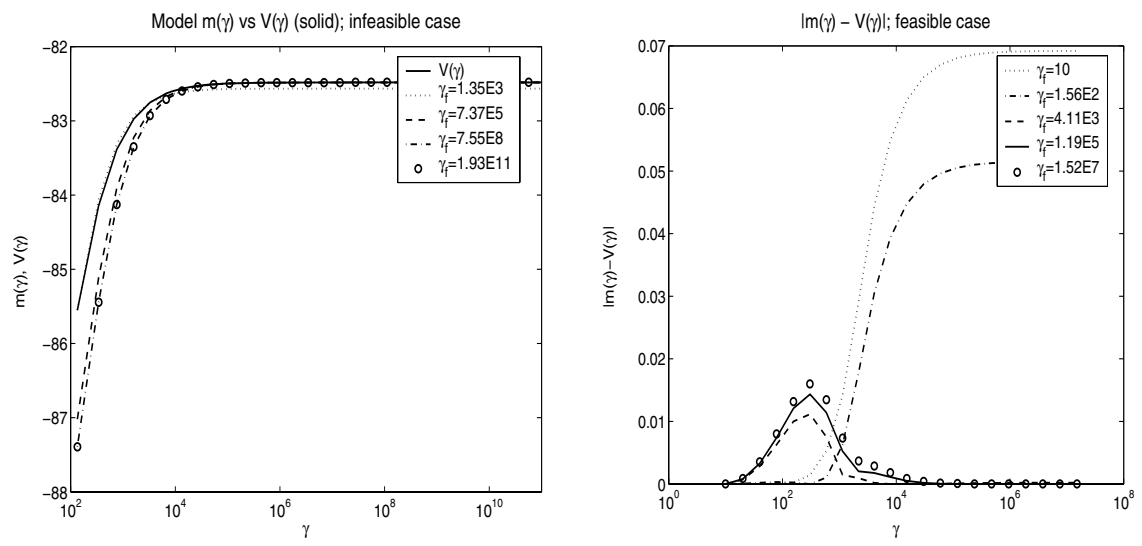

Fig. 4. Left: Model $m(\gamma)$ vs. $V(\gamma)$ (solid) in the infeasible case for P1. Right: Model $m(\gamma)$ vs. $V(\gamma)$ in the feasible case.

by Corollary 4.3 we have $\dot{V}(\gamma)>0$ for all $\gamma>0$. Consequently $V(\gamma)>V(0)$ and $V(\gamma)-V(0)-\gamma \dot{V}(\gamma)=-\int_{0}^{\gamma} \int_{s}^{\gamma} \ddot{V}(\sigma) d \sigma d s>0$, and hence $E>0$ and $C_{2}>0$ for all $\gamma \in(0,+\infty)$. This implies $m(\gamma) \leq C_{1}$ and $m(\gamma) \rightarrow C_{1}$ for $\gamma \rightarrow+\infty$.

We propose the following update strategy for $\gamma$ : Let $\left\{\tau_{k}\right\}$ satisfy $\tau_{k} \in(0,1)$ for all $k \in \mathbb{N}$ and $\tau_{k} \downarrow 0$ as $k \rightarrow \infty$, and assume that $V\left(\gamma_{k}\right)$ is available. Then, given $\gamma_{k}$, the updated value $\gamma_{k+1}$ should ideally satisfy

$$
\left|V^{*}-V\left(\gamma_{k+1}\right)\right| \leq \tau_{k}\left|V^{*}-V\left(\gamma_{k}\right)\right| .
$$

Since $V^{*}$ and $V\left(\gamma_{k+1}\right)$ are unknown, we use $C_{1, k}$ and our model $m_{k}(\gamma)=C_{1, k}-$ $C_{2, k} /\left(E_{k}+\gamma\right)$ at $\gamma=\gamma_{k+1}$ instead. Thus, (44) is replaced by

$$
\left|C_{1, k}-m_{k}\left(\gamma_{k+1}\right)\right| \leq \tau_{k}\left|C_{1, k}-V\left(\gamma_{k}\right)\right|=: \beta_{k} .
$$

Solving the equation $C_{1, k}-m_{k}\left(\gamma_{k+1}\right)=\beta_{k}$, we obtain

$$
\gamma_{k+1}=\frac{C_{2, k}}{\beta_{k}}-E_{k} .
$$

In Theorem 6.1 we shall show that $\gamma_{k+1} \geq \kappa \gamma_{k}$, with $\kappa>1$, independently of $k \in \mathbb{N}$.

Before we turn to the feasible case, we interpret (44) in view of Proposition 4.5 in the infeasible case. Recall that $V^{*} \geq V(\gamma)$, and observe that $\left|V^{*}-V(\gamma)\right|=$ $\mathcal{O}\left(\left|y^{*}-y_{\gamma}\right|_{H_{0}^{1}}\right)$. Proposition 4.5 yields

$$
\left|y^{*}-y_{\gamma}\right|_{H_{0}^{1}}^{2} \leq \frac{2}{\nu}\left(V^{*}-V(\gamma)\right)
$$

since $\dot{V}(\gamma)>0$. Setting $\tau_{k}=\omega_{k}^{2}\left|V^{*}-V\left(\gamma_{k}\right)\right|$, with $\omega_{k} \rightarrow 0$, in (44) yields

$$
\left|y^{*}-y_{\gamma_{k+1}}\right|_{H_{0}^{1}}^{2} \leq C_{\tau} \omega_{k}^{2}\left|y^{*}-y_{\gamma_{k}}\right|_{H_{0}^{1}}^{2} .
$$

Consequently, we obtain

$$
\frac{\left|y^{*}-y_{\gamma_{k+1}}\right|_{H_{0}^{1}}}{\left|y^{*}-y_{\gamma_{k}}\right|_{H_{0}^{1}}} \leq C_{\tau} \omega_{k}
$$


which implies $q$-superlinear convergence of $\left\{y_{\gamma_{k}}\right\}$ in $H_{0}^{1}(\Omega)$.

In the feasible case, i.e., when $\bar{\lambda}$ satisfies (12), we use the model $m(\gamma)=C_{1}-$ $C_{2}(E+\gamma)^{-1}+B \gamma^{-1}$ with $C_{2} \geq 0$ and $E, B>0$; see (37). Let $\gamma_{r}>0, \gamma_{r} \neq \gamma$, denote a reference $\gamma$-value; then we use the conditions

$$
m\left(\gamma_{r}\right)=V\left(\gamma_{r}\right), \quad \dot{m}\left(\gamma_{r}\right)=\dot{V}\left(\gamma_{r}\right), \quad m(\gamma)=V(\gamma), \quad \dot{m}(\gamma)=\dot{V}(\gamma)
$$

for fixing $B, C 1, C 2, E$. Solving the corresponding system of nonlinear equations, we get

$$
E=\frac{\left(\left(\left(\gamma_{r}-\gamma\right)\left(\dot{V}\left(\gamma_{r}\right) \gamma_{r}^{2}+\dot{V}(\gamma) \gamma^{2}\right)+2 \gamma_{r} \gamma\left(V(\gamma)-V\left(\gamma_{r}\right)\right)\right)\right.}{\left(\left(\dot{V}(\gamma) \gamma+\dot{V}\left(\gamma_{r}\right) \gamma_{r}\right)\left(\gamma-\gamma_{r}\right)+\left(\gamma_{r}+\gamma\right)\left(V\left(\gamma_{r}\right)-V(\gamma)\right)\right)}
$$

and

$$
B=\frac{\gamma_{r}^{2} \gamma^{2}\left(\left(V(\gamma)-V\left(\gamma_{r}\right)\right)^{2}-\dot{V}(\gamma) \dot{V}\left(\gamma_{r}\right)\left(\gamma-\gamma_{r}\right)^{2}\right)}{\left(\left(\gamma-\gamma_{r}\right)^{2}\left(\dot{V}\left(\gamma_{r}\right) \gamma_{r}^{2}+\dot{V}(\gamma) \gamma^{2}\right)+2\left(\gamma-\gamma_{r}\right) \gamma_{r} \gamma\left(V\left(\gamma_{r}\right)-V(\gamma)\right)\right)}
$$

Then the parameters $C_{1}$ and $C_{2}$ are given by

$$
\begin{aligned}
C_{2} & =(E+\gamma)^{2}\left(\frac{B}{\gamma^{2}}+\dot{V}(\gamma)\right), \\
C_{1} & =V(\gamma)+\frac{C_{2}}{E+\gamma}-\frac{B}{\gamma} .
\end{aligned}
$$

In the right plot of Figure 4 we show $|m(\gamma)-V(\gamma)|$ with $m(\gamma)$ produced by the iterates of Algorithm EP for P1 similar to the infeasible case. Again we can see that our model yields a close approximation of the value function $V$.

If we require that (45) be satisfied in the feasible case, then we obtain the following update strategy for $\gamma$ :

$$
\gamma_{k+1}=-\frac{D_{k}}{2}+\sqrt{\frac{D_{k}^{2}}{4}+\frac{B_{k} E_{k}}{\beta_{k}}},
$$

where $D_{k}=E_{k}+\left(C_{2, k}-B_{k}\right) / \beta_{k}$. In Theorem 6.1 we shall establish $\gamma_{k+1} \geq \kappa \gamma_{k}$ for all $k \in \mathbb{N}_{0}$ with $\kappa>1$ independent of $k$.

Next we describe an exact path-following version of Algorithm B, which utilizes the update strategy (45) for updating $\gamma$.

Algorithm EP.

(i) Select $\gamma_{r}$. Compute $V\left(\gamma_{r}\right)$, and choose $\gamma_{0}>\max \left(1, \gamma_{r}\right)$; set $k=0$.

(ii) Apply Algorithm B to obtain $y_{\gamma_{k}}$.

(iii) Compute $V\left(\gamma_{k}\right), \dot{V}\left(\gamma_{k}\right)$, and $\gamma_{k+1}$ according to (46) in the infeasible case or (47) in the feasible case.

(iv) Set $k=k+1$, and go to (ii).

Concerning the choice of $\gamma_{r}$ note that in the infeasible case we have $\gamma_{r} \geq 0$, and in the feasible case $\gamma_{r}>0$. Convergence of Algorithm EP is addressed next.

TheOREM 6.1. Assume that the solution to $(\hat{\mathrm{P}})$ is not feasible for $(\mathrm{P})$. Then the iterates $\gamma_{k}$ of Algorithm EP tend to $\infty$ as $k \rightarrow \infty$, and consequently $\lim _{k \rightarrow \infty}\left(y_{\gamma_{k}}, \lambda_{\gamma_{k}}\right)=$ $\left(y^{*}, \lambda^{*}\right)$ in $H_{0}^{1}(\Omega) \times H^{-1}(\Omega)_{\text {weak }}$. 
Proof. Let us consider the infeasible case. Then (45) is equivalent to

$$
0<C_{1, k}-m_{k}\left(\gamma_{k+1}\right)<\tau_{k}\left(C_{1, k}-m_{k}\left(\gamma_{k}\right)\right) .
$$

Since $\gamma \mapsto m_{k}(\gamma)$ is strictly increasing and $\tau_{k} \in(0,1)$, it follows that $\gamma_{k+1}>\gamma_{k}$ for every $k=0,1, \ldots$ If $\lim _{k \rightarrow \infty} \gamma_{k}=\infty$, then $\lim _{k \rightarrow \infty}\left(y_{\gamma_{k}}, \lambda_{\gamma_{k}}\right)=\left(y^{*}, \lambda^{*}\right)$. Otherwise there exists $\bar{\gamma}$ such that $\lim _{k \rightarrow \infty} \gamma_{k}=\bar{\gamma}$. Since $\gamma \mapsto V(\gamma)$ and $\gamma \mapsto \dot{V}(\gamma)$ are continuous on $(0, \infty)$, it follows from (42) and (43) that $\lim _{k \rightarrow \infty} E_{k}=E(\bar{\gamma}), \lim _{k \rightarrow \infty} C_{1, k}=$ $C_{1}(\bar{\gamma})$, and $\lim _{k \rightarrow \infty} C_{2, k}=C_{2}(\bar{\gamma})$, where $E(\bar{\gamma}), C_{1}(\bar{\gamma}), C_{2}(\bar{\gamma})$ are given by $(43)$ with $\gamma$ replaced by $\bar{\gamma}$. Taking the limit with respect to $k$ in (48), we arrive at

$$
\frac{C_{2}(\bar{\gamma})}{E(\bar{\gamma})+\bar{\gamma}}=0
$$

which is impossible, since $C_{2}(\bar{\gamma})>0$ and $E(\bar{\gamma})>0$ if the solution to $(\hat{\mathrm{P}})$ is not feasible for (P). Thus $\lim _{k \rightarrow \infty} \gamma_{k}=\infty$. The feasible case is treated analogously.

Numerically we stop the algorithm as soon as $\left\|\left(r_{k}^{1, h}, r_{k}^{2, h}, r_{k}^{3, h}\right)^{\top}\right\|_{2} \leq \sqrt{\epsilon_{M}}$, where

$$
\begin{aligned}
r_{k}^{1, h} & =\left\|y_{\gamma_{k}}^{h}+\left(-\Delta^{h}\right)^{-1}\left(\lambda_{\gamma_{k}}^{h}-f^{h}\right)\right\|_{H^{-1, h}} /\left\|f^{h}\right\|_{H^{-1, h}}, \\
r_{k}^{2, h} & =\left\|\lambda_{\gamma_{k}}^{h}-\max \left(0, \lambda_{\gamma_{k}}^{h}+y_{\gamma_{k}}^{h}-\psi^{h}\right)\right\|_{H^{-1, h}}, \\
r_{k}^{3, h} & =\left\|\max \left(0, y_{\gamma_{k}}^{h}-\psi^{h}\right)\right\|_{L_{2}^{h}},
\end{aligned}
$$

and $\epsilon_{M}$ denotes the machine accuracy. Here $|\cdot|_{H^{-1, h}}$ denotes the discrete version of $|\cdot|_{H^{-1}}$. For some vector $v$ it is realized as $|v|_{H^{-1}}=\left|\nabla^{h}\left(-\Delta^{h}\right)^{-1} v\right|_{L_{2}^{h}}$ with $|\cdot|_{L_{2}^{h}}$ the discrete $L^{2}$-norm and $\nabla^{h}$ a forward difference approximation of the gradient operator; see [8]. The inner iteration, i.e., Algorithm B for $\gamma=\gamma^{k}$, is terminated if successive active sets coincide or

$$
\frac{\left\|-\Delta^{h} y_{\gamma_{k}}^{h, l}+\lambda_{\gamma_{k}}^{h, l}-f^{h}\right\|_{H^{-1, h}}}{\left\|f^{h}\right\|_{H^{-1, h}}} \leq \sqrt{\epsilon_{M}} .
$$

Here the superscript $l=l(k)$ denotes the iteration index of Algorithm B for fixed $k$. For a discussion and numerical results in the case where the approximation errors due to the discretization of the underlying function space problems are incorporated into the algorithmic framework, e.g., when stopping the algorithm, we refer to the next section 6.2.

The initialization of $\gamma$ is as follows: In the infeasible case we propose a choice of $\gamma_{0}$ based on the deviation of the linearization of $V(\gamma)$ at $\gamma=\gamma_{r}$ from the objective value of the unconstrained problem $(\hat{\mathrm{P}})$ at the projection of $y_{\gamma_{r}}$ onto the feasible set. In our realization of this heuristic we choose $\gamma_{r}=0$ and compute $\hat{y}, V(0)$, and $\dot{V}(0)$. Then we set

$$
\gamma_{0}=\max \left\{1, \zeta \frac{J\left(y_{b}\right)-V(0)}{\dot{V}(0)}\right\},
$$

where $\zeta \in(0,1]$ is some fixed constant, $y_{b}(x)=\min (\hat{y}, \psi(x))$, and $J$ denotes the objective function of $(\hat{\mathrm{P}})$. Note that $\hat{y}$ is the minimizer of the unconstrained problem $(\hat{\mathrm{P}})$. For the examples below we use $\zeta=1$. In the feasible case we choose a reference value $\gamma_{r}$, e.g., $\gamma_{r}=1$, and solve the path problem $\left(\mathrm{P}_{\gamma}\right)$. Then we choose

$$
\gamma_{0}=\gamma_{r}+\frac{J(\hat{y})-V\left(\gamma_{r}\right)}{\dot{V}\left(\gamma_{r}\right)}
$$


where $\hat{y}$ denotes the minimizer of the discretized unconstrained problem $(\hat{\mathrm{P}})$. If $\hat{y}$ is not feasible for $(\mathrm{P})$, then one has $J(\hat{y})<V\left(\gamma_{r}\right)$ and hence $\gamma_{0}>\gamma_{r}$.

When applied to P1, P2, and P3 for $h=1 / 128$ and with $\tau_{k}=0.01^{k+1}$, we obtain the results shown in Figure 5 and Table 6.1.
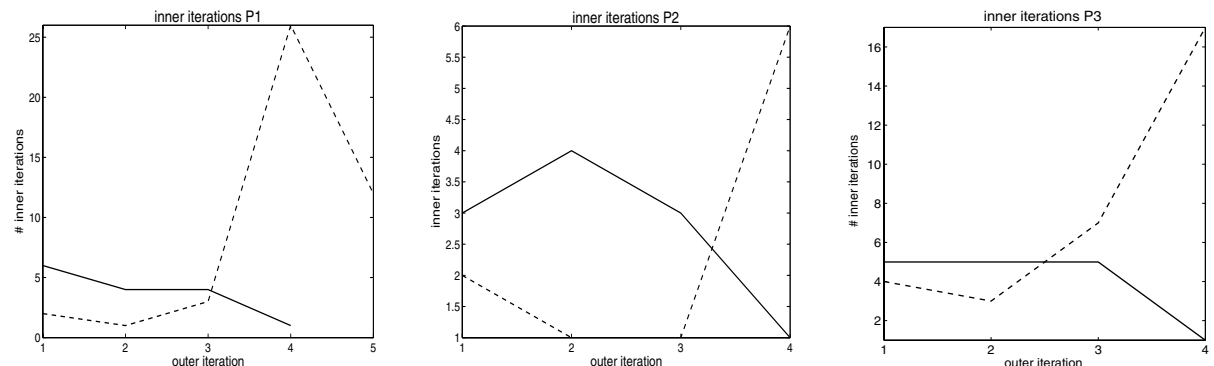

FIG. 5. Number of inner iterations (vertical axis) per outer iteration for P1 (left plot), P2 (middle plot), and P3 (right plot); solid line - infeasible case, dashed line - feasible case.

TABLE 6.1

Comparison of iteration counts.

\begin{tabular}{|c||c|c||c|c|c|c|}
\hline \multicolumn{1}{|c||}{} & \multicolumn{2}{c||}{ P1 } & \multicolumn{2}{c|}{ P2 } & \multicolumn{2}{c|}{ P3 } \\
\hline Version & \# outer & \# inner & \# outer & \# inner & \# outer & \# inner \\
\hline \hline Feasible & 5 & 44 & 4 & 10 & 4 & 31 \\
\hline Infeasible & 4 & 15 & 4 & 11 & 4 & 16 \\
\hline
\end{tabular}

From our test runs, also for other test problems, we observe the following characteristics:

- For the feasible version the number of inner iterations exhibits an increasing tendency until a saturation value is reached, and then, unless the algorithm stops at an approximate solution, it starts to decrease. For the infeasible version we typically observe that the first couple of iterations require several inner iterations. As the outer iterations proceed the number of inner iterations drops eventually to one. We also tested less aggressive $\gamma$-updates compared to the ones used here, e.g., updates based on $\gamma_{k+1}=\xi \gamma_{k}$ with $\xi>1$ fixed.

- The numerically observable convergence speed of $y_{\gamma_{k}}$ towards $y^{*}$ in $H_{0}^{1}(\Omega)$ is typically superlinear. This can be seen from Figure 6, where the plots for the discrete versions $q_{k}^{h}$ of the quotients

$$
q_{k}=\frac{\left|y_{\gamma_{k+1}}-y^{*}\right|_{H_{0}^{1}}}{\left|y_{\gamma_{k}}-y^{*}\right|_{H_{0}^{1}}}
$$

are shown. Note that the vertical axis uses a logarithmic scale. In the first row, for P1 we depict the behavior of $q_{k}^{h}$ for $h=2^{-i}, i=5,6,7,8$, for the infeasible case (left plot) and the feasible case (right plot). We observe that the convergence rate is stable with respect to decreasing mesh size $h$. In the second row we see the behavior of $q_{k}^{h}$ for P2 and P3, with $h=2^{-7}$. Again, we observe a superlinear rate of convergence. With respect to decreasing $h$ the same conclusion as for $\mathrm{P} 1$ holds true. These stability results provide a link between our function space theory and the numerical realization of the algorithms. 

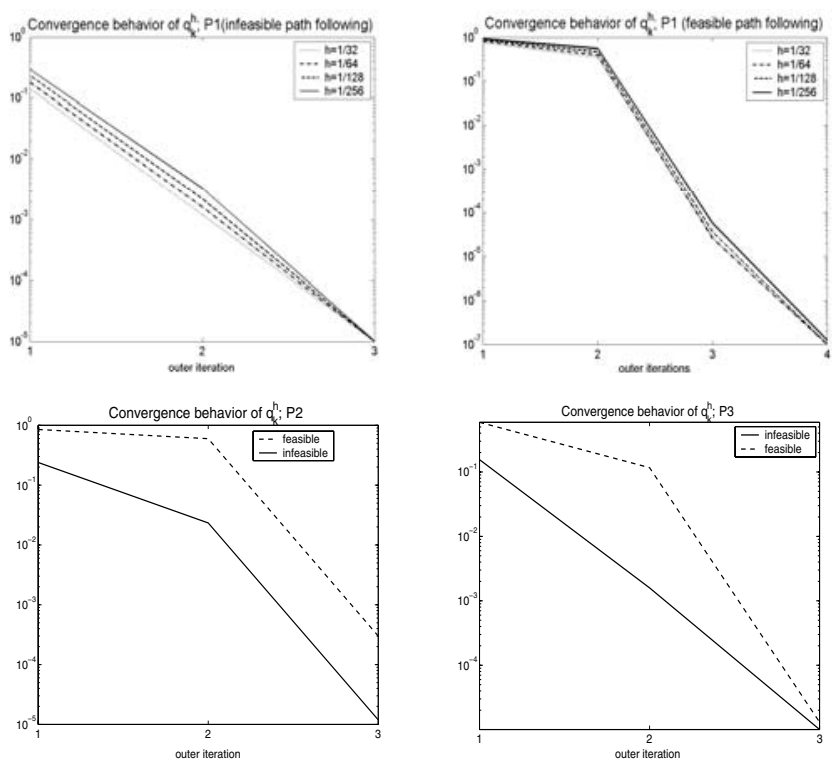

FIG. 6. Discrete quotients $q_{k}^{h}$ for $\mathrm{P} 1$ and various mesh sizes $h$ (upper row) and for $\mathrm{P} 2$ (lower left) and P3 (lower right) for $h=1 / 128$.
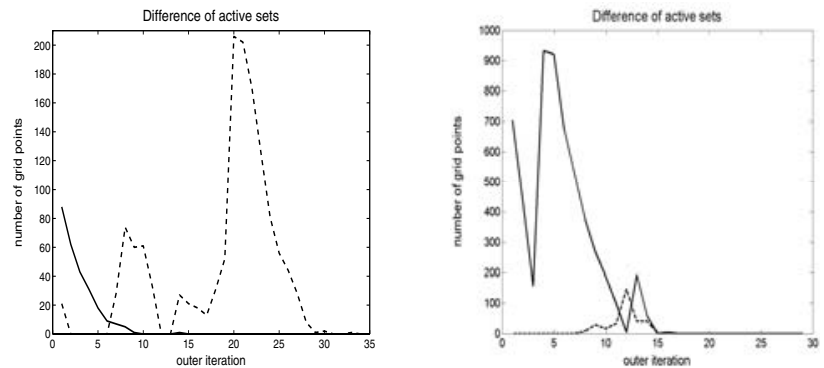

FIG. 7. Difference in active sets for $\mathrm{P} 1$ and $\mathrm{P} 2$; solid line - infeasible case, dashed line - feasible case.

- In connection with the convergence speed it is of interest how the detection process of the correct active set works. For the rather aggressive $\gamma$-updates used in Algorithm EP the difference between two successive active sets is zero typically only in the last iteration. However, if a less aggressive strategy for updating $\gamma$ is used, then it is to be expected, that the difference of active sets might become zero earlier along the iteration. In Figure 7, for the strategy $\gamma_{k+1}=2 \gamma_{k}$, we show the difference of successive active sets; i.e., the vertical axis relates to the number of grid points that are in $\mathcal{A}_{k+1}$ but not in $\mathcal{A}_{k}$ and vice versa. We detect that for the infeasible case there exists an iteration index $\bar{k}$ after which the difference is constantly zero. This behavior is a strong indication that the correct active set was detected. It suggests that we fix this set $\mathcal{A}_{\bar{k}}$ and set $\bar{y}_{\mid \mathcal{A}_{\bar{k}}}=\psi_{\mid \mathcal{A}_{\bar{k}}}, \mathcal{I}_{\bar{k}}=\Omega \backslash \mathcal{A}_{\bar{k}}$, and $\bar{\lambda}_{\mathcal{I}_{\bar{k}}}=0$. Then one computes $\bar{y}_{\mid \mathcal{I}_{\bar{k}}}$ and $\bar{\lambda}_{\mathcal{A}_{\bar{k}}}$ such that $a(\bar{y}, v)+\langle\bar{\lambda}, v\rangle_{H^{-1}, H_{0}^{1}}=(f, v)$ for all $v \in H_{0}^{1}(\Omega)$, and checks whether $(\bar{y}, \bar{\lambda})$ satisfies (7). If this is the case, then the solution is 
found; otherwise $\gamma_{\bar{k}}$ is updated and the iteration continued. If we apply this technique for $\mathrm{P} 1$ in the infeasible case, then the algorithm stops at iteration 15 (35 inner iterations) with the exact discrete solution, as compared to 28 outer and 47 inner iterations without the additional stopping rule. There were four iterations where the additional system solve was necessary but without obtaining the numerical solution. Hence, with respect to system solves, the amount of work drops from 47 solves to $39(=35+4)$. A similar observation is true for P2 and P3. In the feasible case, however, this strategy yields no reduction of iterations. Here, typically the correct active set is determined in the last iteration (for large enough $\gamma$ ).

- The dependence of the iteration number on the mesh size of the discretization for P1 is depicted in Table 6.2 (those for P2 and P3 are similar). In parenthesis we show the number of inner iterations. The results clearly indicate that the outer iterations are mesh independent, while the number of inner iterations increases as the mesh size decreases. In the third row we display the results obtained by applying Algorithm A for the solution of the unregularized problem $(\mathrm{P})$ with data according to $\mathrm{P} 1$. If we compare these results with those of the infeasible exact path-following algorithm, we find that for sufficiently small mesh sizes $h$ the infeasible version of Algorithm EP requires significantly fewer iterations than does Algorithm A, which is also an infeasible algorithm. Also, the number of iterations required by Algorithm A exhibits a relatively strong dependence on $h$ when compared to Algorithm EP in the infeasible case. Similar observations apply also to P2 and P3. This shows that taking into account the function space theoretic properties when regularizing problem $(\mathrm{P})$ results in an algorithmic framework which performs stably with respect to decreasing mesh size of the discretization.

TABLE 6.2

Comparison of iteration counts for different mesh sizes.

\begin{tabular}{|l||c|c|c|c|c|}
\hline \multicolumn{1}{|c||}{} & \multicolumn{5}{c|}{ Mesh size $h$} \\
\hline Version & $1 / 16$ & $1 / 32$ & $1 / 64$ & $1 / 128$ & $1 / 256$ \\
\hline \hline EP feasible & $5(19)$ & $5(23)$ & $5(30)$ & $5(44)$ & $5(72)$ \\
\hline EP infeasible & $4(8)$ & $4(11)$ & $4(13)$ & $4(15)$ & $4(19)$ \\
\hline \hline Algorithm A & 4 & 8 & 14 & 26 & 48 \\
\hline
\end{tabular}
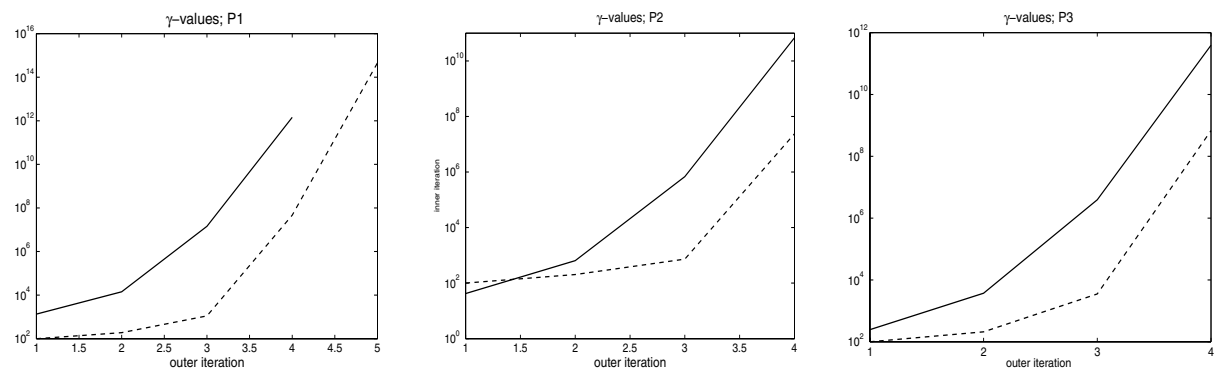

FIG. 8. $\gamma$-updates; solid line - infeasible case, dashed line - feasible case.

- From the plots in Figure 8, where the $y$-axis again has a logarithmic scale, it can be seen that our strategy (45) produces a rapidly increasing sequence 
$\left\{\gamma_{k}\right\}$. The plots in Figure 8 depict the increase of $\gamma_{k}$ as a function of the iteration number. The question arises of whether one could increase $\gamma$ more rapidly. Numerical examples employing an ad hoc strategy show that if $\gamma$ is increased too quickly, then the numerical error may prevent the residuals of the first order system from dropping below $\sqrt{\epsilon_{M}}$. This effect is due to the ill-conditioning of the linear systems for large $\gamma$. On the other hand, small increases in $\gamma$ result in a slow convergence speed of Algorithm EP. Further, in our test runs and as can be seen from Figure 8, the feasible version of Algorithm EP is less aggressive in enlarging $\gamma_{k}$.

6.2. Inexact path-following. While exact path-following is primarily of theoretical interest, the development of inexact path-following techniques that keep the number of iterations as small as possible is of more practical importance. The strategy in the previous section relies on the fact that for every $\gamma_{k}$ the corresponding point on the primal-dual path is computed. This, however, is not the case for inexact techniques and, as a consequence, a different update strategy for the path parameter $\gamma$ is necessary. A common concept in inexact path-following methods is based on the definition of an appropriate neighborhood of the path; see, e.g., [3] and the references therein for a noninterior neighborhood-based path-following method, or [5, 16, 18, 19] for path-following techniques related to interior point methods. It is typically required that the primal-dual iterates stay within the neighborhood of the path, with the goal to reduce the computational burden while still maintaining convergence of the method.

We define

$$
\begin{aligned}
& r_{\gamma}^{1}(y, \lambda)=\|-\Delta y+\lambda-f\|_{H^{-1}}, \\
& r_{\gamma}^{2}(y, \lambda)=\|\lambda-\max (0, \lambda+\gamma(y-\psi))\|_{H^{-1}},
\end{aligned}
$$

and the neighborhood

$$
\mathcal{N}(\gamma):=\left\{(y, \lambda) \in H_{0}^{1}(\Omega) \times L^{2}(\Omega):\left\|\left(r_{\gamma}^{1}(y, \lambda), r_{\gamma}^{2}(y, \lambda)\right)^{\top}\right\|_{2} \leq \frac{\tau}{\sqrt{\gamma}}\right\}
$$

in the infeasible case and

$$
\begin{gathered}
\mathcal{N}(\gamma):=\left\{(y, \lambda) \in H_{0}^{1}(\Omega) \times L^{2}(\Omega):\left\|\left(r_{\gamma}^{1}(y, \lambda), r_{\gamma}^{2}(y, \lambda)\right)^{\top}\right\|_{2} \leq \frac{\tau}{\sqrt{\gamma}}\right. \\
\left.\wedge \frac{\partial}{\partial \gamma} J(y ; \gamma) \leq 0\right\}
\end{gathered}
$$

in the feasible case. Above, $\tau>0$ denotes some fixed parameter. Note that adding the condition $\frac{\partial}{\partial \gamma} J(y ; \gamma) \geq 0$ in (52) yields no further restriction, since this condition is automatically satisfied by the structure of $J(y ; \gamma)$. We also point out that the conditions on the derivative of $J(y ; \gamma)$ are included in (52) and (53), respectively, in order to qualitatively capture (up to first order) the analytical properties of the primal-dual path.

Next we specify our framework for an inexact path-following algorithm.

Algorithm IP.

(i) Initialize $\gamma_{0}$ according to (49) in the infeasible case or (50) in the feasible case; set $k:=0$. 
(ii) Apply Algorithm B to find $\left(y_{k+1}, \lambda_{k+1}\right) \in \mathcal{N}\left(\gamma_{k}\right)$.

(iii) Update $\gamma_{k}$ to obtain $\gamma_{k+1}$.

(iv) Set $k=k+1$, and go to (ii).

Note that if in step (ii) the path-problem $\left(\mathrm{P}_{\gamma}\right)$ is solved, then $r_{\gamma}^{1}\left(y_{\gamma}, \lambda_{\gamma}\right)=r_{\gamma}^{2}\left(y_{\gamma}, \lambda_{\gamma}\right)=$ 0 .

As is the case with primal-dual path-following interior point methods, the update strategy for $\gamma$ in step (iii) of Algorithm IP is a delicate issue. If the increase of $\gamma$ from one iteration to the next is rather small, then we follow the path closely, and the convergence speed is slow. If the $\gamma$-update is too aggressive, then step (ii) requires many iterations of Algorithm B to produce iterates in the neighborhood. We propose the following strategy, which performed very well in our numerical tests.

We introduce the primal infeasibility measure $\rho^{F}$ and the complementarity measure $\rho^{C}$ as follows:

$$
\begin{aligned}
\rho_{k+1}^{F} & :=\int_{\Omega}\left(y_{k+1}-\psi\right)^{+} d x, \\
\rho_{k+1}^{C} & :=\int_{\mathcal{I}_{k+1}}\left(y_{k+1}-\psi\right)^{+} d x+\int_{\mathcal{A}_{k+1}}\left(y_{k+1}-\psi\right)^{-} d x,
\end{aligned}
$$

where $(\cdot)^{-}=-\min (0, \cdot)$ and $(\cdot)^{+}=\max (0, \cdot)$. Note that at the optimal solution both measures vanish. Further, we point out that $\rho^{C}$ is related to the duality measure well known from primal-dual path-following interior point methods. These measures are used in the following criterion for updating $\gamma$ :

$$
\gamma_{k+1} \geq \max \left(\gamma_{k} \max \left(\tau_{1}, \frac{\rho_{k+1}^{F}}{\rho_{k+1}^{C}}\right), \frac{1}{\left(\max \left(\rho_{k+1}^{F}, \rho_{k+1}^{C}\right)\right)^{q}}\right)
$$

with $\tau_{1}>1$ and $q \geq 1$. The first term in the outermost max-expression is used because of our observation that $\rho_{k+1}^{F} \geq \rho_{k+1}^{C}$ in the infeasible case. If $\rho^{C}$ is small compared to $\rho^{F}$, we find that the iterates primarily lack feasibility as compared to complementarity. Therefore, a strong increase in $\gamma$, which aims at reducing constraint infeasibility, is favorable. If both measures are of almost the same size and rather small, then the second term in the outer max-expression should yield a significant increase in $\gamma$. Typically $q \in\left[\frac{3}{2}, 2\right]$ is chosen, which induces growth rates for $\gamma$.

If there is still a significant change in the active sets from one iteration to the next and the update $\gamma_{k+1}$ based on (56) would be too large compared to $\gamma_{k}$, then many inner iterations would be necessary to keep track of the path, or very conservative $\gamma$-updates in the following iterations have to be chosen. We safeguard the $\gamma$-updates by utilizing our model function $m(\gamma)$, which was found to be a reliable tool. In fact, in updating $\gamma$, large deviations from $m(\gamma)$ are prohibited by comparing the value of the tangent to $J(y ; \gamma)$ at $\gamma=\gamma_{k}$ with the actual model value. If necessary and as long as $\gamma_{k+1}$ is much larger than $\gamma_{k}$, we reduce the actual $\gamma$-value until

$$
\left|t_{k}\left(\gamma_{k+1}\right)-m_{k}\left(\gamma_{k+1}\right)\right| \leq \tau_{3}\left|J\left(y_{k+1} ; \gamma_{k}\right)-J\left(y_{k} ; \gamma_{k-1}\right)\right|
$$

with $0<\tau_{3}<1, t_{k}(\gamma)=J\left(y_{k+1} ; \gamma_{k}\right)+\frac{\partial J}{\partial \gamma}\left(y_{k+1} ; \gamma_{k}\right)\left(\gamma-\gamma_{k}\right)$, and $m_{k}(\gamma)$ the model related to $\gamma_{k}$. Recall that $m_{k}\left(\gamma_{k}\right)=J\left(y_{k+1} ; \gamma_{k}\right)$. The motivation of this strategy utilizes the good approximation qualities of our models. Indeed, for small $\gamma$ the distance between $t_{k}$ and $m_{k}$ might be large, but so is $\left|J\left(y_{k+1} ; \gamma_{k}\right)-J\left(y_{k} ; \gamma_{k-1}\right)\right|$ since the change in the function value is expected to be relatively large for small $\gamma$. For large $\gamma$, however, both difference measures tend to be small. 
Concerning the numerical realization of Algorithm IP in the discrete setting we point out that by an a posteriori analysis of the discretization errors one finds that the norm of the residuals in (51a) and (51b) can be approximated typically to the order of $h$. This can be used as an upper bound for $\gamma$ in the discrete versions of (52) and (53), respectively. However, since, on a fixed grid, our discrete versions of (P) and $\left(\mathrm{P}_{\gamma}\right)$ are consistent $($ as $\gamma \rightarrow \infty)$ and admit unique solutions in $\mathbb{R}^{N_{h}}$, where $N_{h} \in \mathbb{N}$ depends on the mesh size of discretization $h$, it is of interest to consider $\gamma \rightarrow \infty$. On a fixed grid, this allows us also to study the behavior of our discretized algorithms as finite dimensional solvers for problems similar to the discrete versions of the ones under consideration. With respect to the latter aspect, below we report on test runs of Algorithm IP when applied to our test problems P1, P2, and P3. The parameters had values $q=1.5, \tau_{1}=10, \tau_{3}=0.999, \tau=1 e 6$. The stopping rule for the outer iteration is as before.

P1. The infeasible version of Algorithm IP requires 9 outer iterations and at most 2 inner iterations per outer iteration. In particular, in many iterations the criterion $\left(y_{k+1}, \lambda_{k+1}\right) \in \mathcal{N}\left(\gamma_{k}\right)$ was satisfied within 1 inner iteration. The feasible version of Algorithm IP stops after 11 iterations. With respect to inner iterations in the feasible case we note that more than 1 or 2 inner iterations were necessary only in the last 3 outer iterations with 3,4 , and 6 inner iterations, respectively. For both runs, the behavior of the measures $\rho^{F}$ and $\rho^{C}$ is shown in Figure 9. Note that the vertical scale is a logarithmic one. The left plot corresponds to the infeasible case. The feasibility measure $\rho^{F}$ and the complementarity measure $\rho^{C}$ are both convergent at a superlinear rate. In the feasible case, which is depicted in the right plot, we observe that $\rho^{C}$ is only linearly convergent. In some iterations we have $\rho_{k}^{F}>0$. However, the constraint violation is of the order of the machine precision and thus negligible.
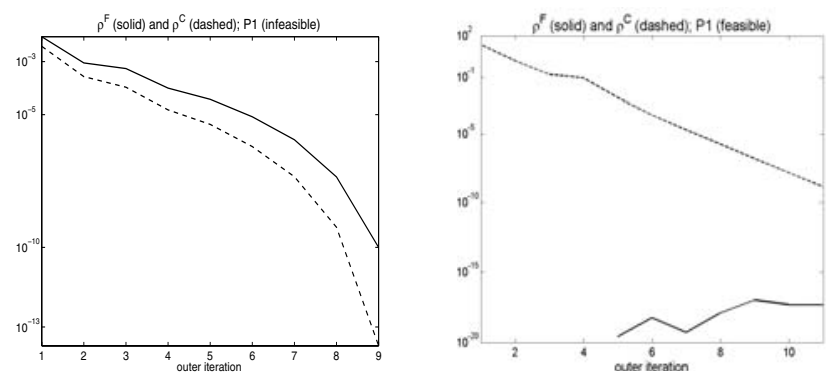

FIG. 9. Behavior of the measures $\rho^{F}$ (solid) and $\rho^{C}$ (dashed) for P1, left plot-infeasible case, right plot-feasible case.

P2. For this test problem the infeasible version of Algorithm IP required 11 iterations with one inner iteration per outer iteration. The feasible version needed 6 outer iterations and 9 inner iterations.

P3. The behavior of Algorithm IP for solving P3 is comparable to its behavior for P1 and P2. In fact, the infeasible version required 11 outer iterations and 11 inner iterations for solving the discrete problem. The feasible variant of Algorithm IP stopped successfully after 9 outer and 19 inner iterations. For the latter run, in the next-to-last iteration 5 inner iterations were necessary; otherwise at most 2 inner iterations were needed. With respect to the behavior of the decrease of the measures $\rho^{C}$ and $\rho^{F}$, an observation similar to the one obtained from Figure 9 for P1 holds true. We remark only that in the feasible case $\rho^{C}$ exhibits an almost superlinear 
convergence behavior.

Compared to the exact path-following strategy of Algorithm EP, the inexact pathfollowing concept of Algorithm IP is in many cases more efficient. In Table 6.3 we provide the number of outer and inner iterations for exact versus inexact path-following. In parenthesis we write the number of inner iterations.

TABLE 6.3

Comparison of iteration counts between exact and inexact path-following.

\begin{tabular}{|c|c|c|c||c|c|c|}
\hline & \multicolumn{3}{|c||}{ Infeasible case } & \multicolumn{3}{c|}{ Feasible case } \\
\hline & P1 & P2 & P3 & P1 & P2 & P3 \\
\hline \hline EP & $4(15)$ & $4(11)$ & $4(16)$ & $5(44)$ & $4(10)$ & $4(31)$ \\
\hline IP & $9(12)$ & $11(11)$ & $11(11)$ & $11(25)$ & $6(9)$ & $9(19)$ \\
\hline
\end{tabular}

Finally we address the issue of how to incorporate the approximation error due to the discretization of function space quantities; see [6, 7]. First note that with (8) holding (which is the case for P3), the discretization of the residual in the definition of the neighborhoods (52), respectively (53), approximates the original one to the order of $h$. Hence, in our discrete version of Algorithm IP the neighborhood criterion

$$
\left\|\left(r_{\gamma}^{1}(y, \lambda), r_{\gamma}^{2}(y, \lambda)\right)^{\top}\right\|_{2} \leq \frac{\tau}{\sqrt{\gamma}}
$$

becomes

$$
\left\|\left(r_{\gamma}^{1, h}(y, \lambda), r_{\gamma}^{2, h}(y, \lambda)\right)^{\top}\right\|_{2} \leq \max \left\{\sqrt{\epsilon_{M}}, \kappa_{\text {in }} h, \frac{\tau}{\sqrt{\gamma}}\right\}
$$

with some constant $\kappa_{\text {in }}>0$. We stop the outer iteration as soon as the discrete residual drops below $\max \left\{\kappa_{\text {out }} h, \sqrt{\epsilon_{M}}\right\}$, where $\kappa_{\text {out }}>0$ is fixed. In our tests we use $\kappa_{\text {in }}=1$ and $\kappa_{\text {out }}=10$. Applying this strategy for the solution of P3, we obtain (outer) iteration numbers as displayed in Table 6.4. Here, in parenthesis we give the total number of inner iterations.

TABLE 6.4

Inexact path-following with h-dependent stopping of inner and outer iterations.

\begin{tabular}{|c||c|c|c|c|c|c|}
\hline \multicolumn{1}{|c||}{ Version } & \multicolumn{6}{|c|}{ Mesh size } \\
\hline & $1 / 16$ & $1 / 32$ & $1 / 64$ & $1 / 128$ & $1 / 256$ & $1 / 512$ \\
\hline IP & $1(1)$ & $4(4)$ & $5(5)$ & $8(8)$ & $9(10)$ & $10(10)$ \\
\hline
\end{tabular}

\section{REFERENCES}

[1] M. Bergounioux, M. Haddou, M. Hintermüller, and K. Kunisch, A comparison of a Moreau-Yosida-based active set strategy and interior point methods for constrained optimal control problems, SIAM J. Optim., 11 (2000), pp. 495-521.

[2] S. C. Brenner and L. R. Scott, The Mathematical Theory of Finite Element Methods, 2nd ed., Texts in Appl. Math. 15, Springer-Verlag, New York, 2002.

[3] X. Chen and P. Tseng, Noninterior continuation methods for solving semidefinite complementarity problems, Math. Program. Ser. A, 95 (2003), pp. 431-474.

[4] A. V. Fiacco And G. P. McCormick, Nonlinear Programming, Sequential Unconstrained Minimization Techniques, Classics in Appl. Math. 4, SIAM, Philadelphia, PA, 1990.

[5] A. Forsgren, P. E. Gill, AND M. H. Wright, Interior methods for nonlinear optimization, SIAM Rev., 44 (2002), pp. 525-597. 
[6] C. Grossmann and A. A. Kaplan, On the solution of discretized obstacle problems by an adapted penalty method, Computing, 35 (1985), pp. 295-306.

[7] C. Grossmann and H.-G. Roos, Numerik partieller Differentialgleichungen, 2nd ed., Teubner Studienbücher Mathematik, B. G. Teubner, Stuttgart, 1994.

[8] W. HackBusch, Theorie und Numerik elliptischer Differentialgleichungen, Teubner Verlag, Stuttgart, 1986.

[9] M. HintermüLler, Inverse coefficient problems for variational inequalities: Optimality conditions and numerical realization, M2AN Math. Model. Numer. Anal., 35 (2001), pp. 129 152.

[10] M. HintermüLler, K. Ito, And K. Kunisch, The primal-dual active set strategy as a semi smooth Newton method, SIAM J. Optim., 13 (2003), pp. 865-888.

[11] K. Ito And K. Kunisch, Optimal control of elliptic variational inequalities, Appl. Math. Optim., 41 (2000), pp. 343-364.

[12] K. Ito AND K. KUNISCH, Semismooth Newton methods for state-constrained optimal control problems, Systems Control Lett., 50 (2003), pp. 221-228.

[13] K. Ito AND K. Kunisch, Semismooth Newton methods for variational inequalities of the first kind, M2AN Math. Model. Numer. Anal., 37 (2003), pp. 41-62.

[14] G. M. Troianiello, Elliptic Differential Equations and Obstacle Problems, Univ. Ser. Math., Plenum Press, New York, 1987.

[15] M. UlbRICH, Semismooth Newton methods for operator equations in function spaces, SIAM J. Optim., 13 (2003), pp. 805-842.

[16] R. J. Vanderbei, Linear Programming, Foundations, and Extensions, 2nd ed., Internat. Ser. Oper. Res. Management Sci. 37, Kluwer Academic Publishers, Boston, MA, 2001.

[17] M. WeISER, Interior point methods in function space, SIAM J. Control Optim., 44 (2005), pp. 1766-1786.

[18] S. J. Wright, Primal-dual Interior-Point Methods, SIAM, Philadelphia, PA, 1997.

[19] Y. Ye, Interior Point Algorithms, Theory, and Analysis, Wiley-Interscience, New York, 1997. 